\title{
Mathematical Models with Exact Renormalization for Turbulent Transport
}

\author{
Marco Avellaneda ${ }^{1, \star}$ and Andrew J. Majda ${ }^{2, \star \star}$ \\ ${ }^{1}$ Courant Institute, New York University, New York, NY 10012, USA \\ ${ }^{2}$ Department of Mathematics and Program in Applied and Computational Mathematics, \\ Princeton University, Princeton, NJ 08544, USA
}

\begin{abstract}
The advection-diffusion of a passive scalar by incompressible velocity fields which admit a statistical description and involve a continuous range of excited spatial and/or temporal scales is very important in applications ranging from fully developed turbulence to the diffusion of tracers in heterogeneous porous media. A variety of renormalization theories which typically utilize partial resummation of divergent perturbation series according to various recipes have been applied to this problem in various contexts. In this paper, a simple model problem for the advection-diffusion of a passive scalar is introduced and the complete renormalization theory is developed with full mathematical rigor. Explicit formulas for the anomalous time scaling in various regimes as well as the Green's function for the large-scale, long-time, ensemble average are developed here. Formulas for the renormalized higher order statistics are also developed. The simple form of the model problem is deceptive; the renormalization theory for this problem exhibits a remarkable range of different renormalization phenomena as parameters in the velocity statistics are varied. These phenomena include the existence of several distinct anomalous scaling regimes as the spectral parameter $\tilde{\varepsilon}$ is varied as well as explicit regimes in $\tilde{\varepsilon}$ where the effective equation for the ensemble average is not a simple diffusion equation but instead involves an explicit random nonlocal eddy diffusivity. We use Fourier analysis and the Feynman-Kac formula as main tools in the explicit exact renormalization theory developed here.
\end{abstract}

\footnotetext{
* Research partially supported by NSF DMS8802739, ARO DAAL03-89-K-0039

$\star \star$ Research partially supported by NSF DMS8702864, ARO DAAL03-89-K-0013, ONR N00014-89-J-1044
} 


\section{Introduction}

The advection-diffusion of a passive scalar by an incompressible velocity field is given by the equation

$$
\begin{gathered}
\frac{\partial T}{\partial t}+(v \cdot \nabla) T=\frac{1}{2} v_{0} \Delta T, \\
\left.T\right|_{t=0}=T_{0}(x),
\end{gathered}
$$

where the incompressible velocity field, $v(x, t)$ satisfies $\operatorname{div} v=0$ and $v_{0}>0$ is the diffusion coefficient. The problem in (1.1) is especially important and difficult when the velocity, $v$, involves a continuous range of excited space and/or time scales and admits a statistical description. Practical applications where these are the circumstances include predicting temperature profiles in high Reynolds number turbulence ([4]), the tracking of pollutants in the atmosphere ([7]), and the diffusion of tracers in heterogeneous porous media ([13]). The problem in (1.1) with velocity fields having a continuous range of excited scales has been attacked through a wide variety of physical space and/or Fourier space renormalization theories $([15,25,8,24,14,27])$ which typically utilize partial summation of divergent perturbation series according to various recipes.

An extremely important practical problem where such renormalization theories have been applied involves the computation of turbulent diffusivities, i.e. "eddy-viscosities," in fully developed turbulence $([25,16])$. With $L_{0}$, the integral length scale and $\bar{U}$, the typical velocity of the energy containing but non-universal fluid motions, the Reynolds number is given by $\operatorname{Re}=\bar{u} L_{0} / v$ with $v$, the kinematic viscosity; the universal inertial range scales for the velocity statistics occur on length scales smaller than $L_{0}$. The objective of theories of "eddy-viscosity" modelling in the context of (1.1) is to determine an effective equation of motion for $T(x, t)$ varying only on length scales larger than $L_{0}$ for times at least comparable to the large scale by turnover time, $\bar{t}=L_{0} / \bar{U}$. Assuming Kolmogoroff statistics for a steady velocity field $v_{\delta}(x)$, the problem in (1.1) becomes

$$
\begin{gathered}
\frac{\partial T^{\delta}}{\partial t}+v_{\delta} \cdot \nabla T^{\delta}=\frac{1}{2} v_{0} \Delta T^{\delta}, \\
\left.T^{\delta}\right|_{t=0}=T_{0}(\delta x),
\end{gathered}
$$

where $v_{\delta}(x)$ is an incompressible velocity field with energy spectrum

$$
\left\langle\left|\hat{v}_{\delta}(k)\right|^{2}\right\rangle= \begin{cases}|k|^{1-d-5 / 3}, & \delta<|k|<1 \\ 0 & \text { otherwise }\end{cases}
$$

with $d=3$ the space dimension. Here $\delta=(\operatorname{Re})^{-3 / 4}$ and since we are interested in fully developed turbulence, $\operatorname{Re} \lambda \infty$ or equivalently $\delta \downarrow 0$. [The reformulation in (1.3) is discussed completely in Sect. 2 of this paper.] If $t^{\prime}=\varrho^{2}(\delta) t$ is a suitable scaling of time, $T\left(x^{\prime}, t^{\prime}\right)$ is defined by

$$
T\left(x^{\prime}, t^{\prime}\right)=\left\langle T^{\delta}\left(\frac{x^{\prime}}{\delta}, \frac{t^{\prime}}{\varrho^{2}(\delta)}\right)\right\rangle,
$$


where $\langle\cdot\rangle$ denotes the ensemble average over velocity statistics. The goal of "eddy diffusivity" theories for (1.2) is to predict the appropriate scaling function of time, $\varrho^{2}(\delta)$, and a simplified dynamic equation for $T\left(x^{\prime}, t^{\prime}\right)$ which remains uniformly valid as $\operatorname{Re} \nearrow \infty$, i.e. $\delta \downarrow 0$. The subtle difficulty with the problem in (1.2), (1.3) is that there is an infrared (low wave number) divergence of the energy spectrum in (1.3) as $\delta \downarrow 0$ and there is no separation of scales between the low wave numbers, $|k| \leqq \delta$, and the regimes with significant amplitude in the velocity spectrum (see Sect. 2 for further discussion).

The main objective of this paper is to study a model problem which is an extremely special case of (1.1) and is strongly motivated by the problem of determining "eddy diffusivities" with infrared divergence which we describe in the preceding paragraph. The simple form of the model problem is deceptive; this problem exhibits a remarkable range of different renormalization phenomena for the scaling laws, the effective equations, and higher moments as parameters in the velocity statistics are varied. Furthermore, we solve this problem with complete mathematical rigor and find explicit formulas for both the time scaling function $\varrho(\delta)$ and the Green's function for the large scale long-time ensemble average, $\langle T\rangle$. We also determine the statistics of higher moments with complete rigor. Thus, this simple model problem provides a nontrivial but unambiguous test problem for renormalization theories of eddy diffusivity. Elsewhere, the authors (see [2]) use the exact results presented here to study the variety of approximate renormalization theories listed earlier.

The model problem which we study here is the special case of (1.1) given by

$$
\begin{gathered}
\frac{\partial T^{\delta}}{\partial t}+v_{\delta}(x, t) \frac{\partial T^{\delta}}{\partial y}=\frac{1}{2} v_{0} \Delta T^{\delta}, \\
\left.T^{\delta}\right|_{t=0}=T_{0}(\delta x, \delta y) .
\end{gathered}
$$

The incompressible velocity field in (1.4) is a simple shearing motion along the $y$-axis. Motivation for the velocity statistics which we assume for the model problem is provided, for example, by the problem of computing scalings and equations for eddy diffusivity for the problem described in (1.2) and (1.3) above. In the case of steady velocity fields $v_{\delta}(x)$, we assume that $v_{\delta}(x)$ is a stationary Gaussian field with energy spectrum

$$
\left\langle\left|\hat{v}_{\delta}^{S}(k)\right|^{2}\right\rangle=(2 \pi)^{1 / 2}|k|^{1-\tilde{\varepsilon}} \psi_{0}\left(\frac{|k|}{\delta}\right) \psi_{\infty}(|k|)
$$

Here $\tilde{\varepsilon}$ with $-\infty<\tilde{\varepsilon}<\infty$ is a parameter characterizing the velocity spectrum and is familiar from renormalization theory $([17,8,27])$. The functions $\psi_{0}\left(\frac{|k|}{\delta}\right)$ and $\psi_{\infty}(|k|)$ are infrared and ultraviolet cut-offs respectively and correspond to the restrictions, $\delta<|k|<1$, in (1.3). For the time dependent case in (1.4), we assume that $v_{\delta}(x, t)$ has stationary Gaussian statistics with energy-power spectrum given by

$$
\left\langle\left|\hat{v}_{\delta}(k, \omega)\right|^{2}\right\rangle=\left|\hat{v}_{\delta}^{S}(k)\right|^{2}(a|k|)^{-z} \phi\left(\frac{\omega}{a|k|^{2}}\right)
$$


with $\phi(s)=\pi^{-1}\left(1+s^{2}\right)^{-1}$. Here $z$ is a parameter satisfying $0<z<\infty$. The precise assumptions on the velocity statistics in (1.5) and (1.6) are given in Sect. 2 after the motivation of a general discussion of the problem of developing theories for eddy viscosity for incompressible velocity fields with a continuous range of excited space and time scales - the brief discussion in (1.2) and (1.3) is a special case.

In the model problem from (1.4), we seek unique space-time rescalings

$$
x^{\prime}=\delta x, \quad y^{\prime}=\delta y, \quad t^{\prime}=\varrho^{2}(\delta) t
$$

with $\varrho(\delta) \downarrow 0$ as $\delta \downarrow 0$ so that the ensemble average over velocity statistics in the rescaled variables satisfies an appropriate eddy equation, i.e. after dropping the primes in (1.7), the quantity defined by

$$
\bar{T}(x, y, t)=\lim _{\delta \downarrow 0}\left\langle T^{\delta}\left(\frac{x}{\delta}, \frac{y}{\delta}, \frac{t}{\varrho^{2}(\delta)}\right)\right\rangle
$$

satisfies an appropriate "homogenized" effective equation. The model problem in (1.4) with the stationary Gaussian statistics in (1.5) and (1.6) has a special structure which enables us to develop exact representation formulas for the solution $T^{\delta}$ of (1.4) through Fourier analysis and the Feynman-Kac formula. In Sect. 3, we develop general representation and renormalization formulas which enable us to compute rigorous formulas for both the scaling function $\varrho(\delta)$ in (1.7) and $\left\langle T^{\delta}\right\rangle$ as the parameters $\tilde{\varepsilon}$ and $z$ in (1.5) and (1.6) vary. In Sect. 4, we develop the renormalization theory for the steady case with Gaussian velocity statistics and power spectrum from (1.5). The unsteady case with Gaussian velocity statistics and spectrum from (1.6) is discussed in Sect. 5. The limits of higher order statistics are developed in Sect. 6. Several facts regarding the mean field regime are presented in the appendix; in particular, our results on the time-dependent case are not available elsewhere in the literature. Next we summarize the remarkable phenomena that occur in the renormalization theory for (1.4) including explicit random nonlocal eddy diffusivity equations for $\left\langle T^{\delta}\right\rangle$ in some regimes of $\tilde{\varepsilon}$ for appropriate values of $z$, as well as several distinct anomalous scaling regimes as $\tilde{\varepsilon}$ is varied for fixed $z$.

In developing the rigorous renormalization theory for the steady case in (1.4), (1.5), we find three distinct scaling regimes for the model problem. The first regime is defined by the parameter range, $\tilde{\varepsilon}<0$. This regime is a region of mean field theory. In the region $\tilde{\varepsilon}<0$, the energy containing length scales associated with the velocity spectrum in (1.5) are sufficiently separated from the large scales defined by $\delta$ so that the classical formulas of homogenization theory apply (see $[20,23,3]$ ); the time scaling law is the expected diffusive scaling, $\varrho(\delta)=\delta$; the coefficients for the effective diffusion equation are determined by standard formulas and depend on the bare viscosity $v_{0}$ as well as the ultraviolet cut-off $\psi_{\infty}(|k|)$.

The second regime of parameters is determined by $\tilde{\varepsilon}>2$ and corresponds to an anomalous scaling regime dominated by inviscid dynamics. The time scaling function is given by

$$
\varrho(\delta)=\delta^{1-\tilde{\varepsilon} / 4}, \quad 2<\tilde{\varepsilon}<4
$$

and the effective equation for $\left\langle T^{\delta}\right\rangle$ is a simple diffusion equation

$$
\frac{\partial \bar{T}}{\partial t}=D(\tilde{\varepsilon}) t \bar{T}_{y y}
$$


where $D(\tilde{\varepsilon})$ depends on the ultraviolet cut off $\psi_{0}(|k|)$ but is independent of the bare diffusivity. We remark that the scaling $\varrho(\delta)=\delta^{1 / 2}$ corresponds to the expected convective scaling for the "inviscid" problem in (1.4) with $v_{0}=0$ and the time scales in (1.9) A) are "hyperconvective," i.e. faster than the convective scale. In Sect. 4 we show that the renormalization theory for (1.4) for $\tilde{\varepsilon}>2$ in the steady case coincides with the renormalization theory for the "inviscid" problem.

The third regime of renormalization for the steady case is defined by the inequality, $0<\tilde{\varepsilon}<2$. In Sect. 4, we derive the renormalized scaling law

$$
\varrho(\delta)=\delta^{\frac{1}{1+\tilde{\varepsilon} / 2}}, \quad 0<\tilde{\varepsilon}<2 .
$$

The function $\varrho(\delta)$ in (1.10) agrees with the diffusive scaling at $\tilde{\varepsilon}=0$ but is an anomalous scaling intermediate between the diffusive scaling and the purely convective scaling, $\delta^{1 / 2}$, which occurs at $\tilde{\varepsilon}=2$. Thus, from (1.9) A) and (1.10), we observe that there are two distinct anomalous scaling regimes for the model problem with steady velocity fields. With the effective equation in (1.9) B) for $\tilde{\varepsilon}>2$ and the scaling law in (1.10), naively one might guess that the effective equation for $\left\langle T^{\delta}\right\rangle$ is a simple local diffusion equation with the form,

$$
\frac{\partial \bar{T}}{\partial t}=\left(1+\frac{\tilde{\varepsilon}}{2}\right) t^{\varepsilon / 2} \mathscr{D} \alpha \bar{T}_{y y},
$$

where $\mathscr{D}=\left(v_{0}\right)^{\tilde{z} / 2-1}(4 \pi)^{-1}$ and $\alpha$ has some prescribed value, say $\bar{\alpha}(\tilde{\varepsilon})$, depending on $\tilde{\varepsilon}$; the equation in (1.11) has the same form as the equation in (1.9) B) for $\tilde{\varepsilon}=2$ and also has the correct scaling with $t$ at $\tilde{\varepsilon}=0$. This naive guess is wrong!! Let $K(y, t, \alpha)$ denote the explicit Green's function for (1.11) for a fixed value of $\alpha$. Then, for each value of $\tilde{\varepsilon}$ with $0<\tilde{\varepsilon}<2$, there is a distribution function of random diffusivities, $v_{\varepsilon}(\alpha)$, so that the Green's function for the effective equation is given by

$$
K^{\tilde{\varepsilon}}(y, t)=\int_{0^{+}}^{\infty} K(y, t, \alpha) d v_{\tilde{\varepsilon}}(\alpha)
$$

with

$$
\bar{T}(x, y, t)=\int K^{\tilde{\varepsilon}}(y-\tilde{y}, t) T_{0}(x, \tilde{y}) d \tilde{y},
$$

and $T_{0}(x, y)$, the (rescaled) initial data. In particular, since $d v_{\varepsilon}(\alpha)$ is not a point mass, the Green's function for (1.12) does not have a spatial Gaussian profile for $0<\tilde{\varepsilon}<2$ and the effective equation for the eddy viscosity theory is not a simple local diffusion equation. The formulas for the distribution function $v_{\varepsilon}(\alpha)$ for random diffusivity exhibit remarkable changes in complexity as the parameter $\tilde{\varepsilon}$ is varied with $0<\tilde{\varepsilon}<2$ and the interested reader should consult Sect. 4 for the details. Some approximate renormalization theories for eddy diffusivity anticipate non-local effective equations $([14,16])$ but our work on the model problem in Sect. 4 appears to be the first time that explicit formulas for the Green's function have been found without any approximation.

In Sect. 5, we discuss rigorous renormalization for the model problem in (1.4) for time dependent velocity fields with statistics described in (1.6). Figure 1 and Table 1 highlight the remarkable range of different phenomena in renormalization that occur as the spectral parameters $\tilde{\varepsilon}$ and $z$ vary. To gain some intuition into these phenomena, we see from (1.6) (see Sect. 5) that $a|k|^{z}$ measures the correlation 


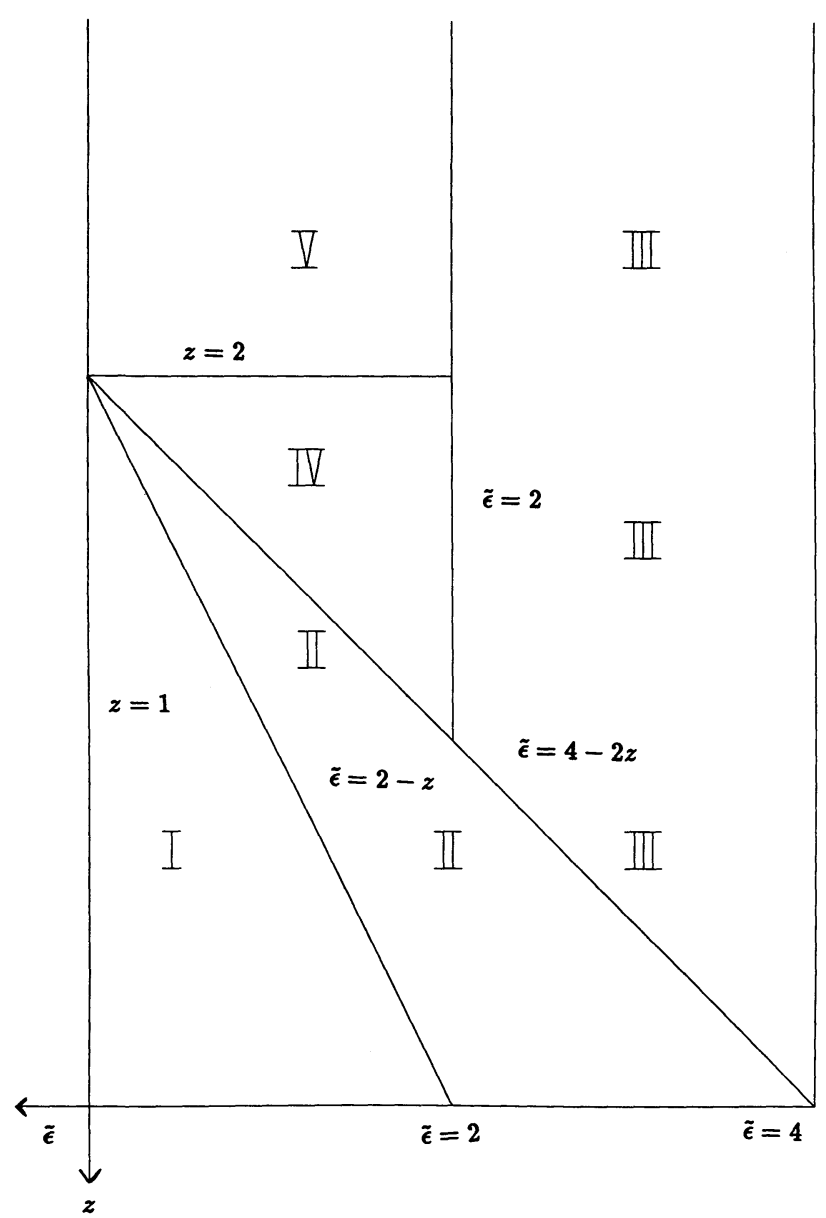

Fig. 1. The five regions in the $(\tilde{\varepsilon}, z)$ upper half plane with different behavior for the renormalization theory

time of the velocity statistics at wave number $k$ while only wave numbers with $|k| \ll 1$ are the significant range for computing effective equations for $\left\langle T^{\delta}\right\rangle$. Thus, the parameter $z$ has the following significance: when $z$ is small there is rapid decorrelation in time at the reelvant wave numbers while stronger correlations in time develop at a fixed low wave number as $z$ increases. This intuition summarizes the trends depicted in Fig. 1 and Table 1. There is an abrupt transition in behavior at $z=2$ in the hyperscaling regimes where $\delta / \varrho(\delta) \downarrow 0$. For $z<2$, there is sufficiently rapid decorrelation in time at low wave numbers so that the renormalization theory for (1.4) coincides in hyperscaling regimes exactly with the renormalization theory for the "inviscid" problem,

$$
\begin{gathered}
\frac{\partial T^{\delta}}{\partial t}+v_{\delta}(x, t) \frac{\partial T^{\delta}}{\partial y}=0, \\
\left.T^{\delta}\right|_{t=0}=T_{0}(\delta x, \delta y)
\end{gathered}
$$


Table 1. Summary of the exact renormalization theory for the five regions

\begin{tabular}{|c|c|c|c|c|c|}
\hline Region & I & II & III & IV & V \\
\hline $\begin{array}{l}\text { Eulerian } \\
\text { scaling law }\end{array}$ & $\begin{array}{l}\varrho_{1}(\delta)=\delta \\
\quad \text { (Meanfield } \\
\text { theory) }\end{array}$ & $\begin{array}{l}\varrho_{2}(\delta)=\delta \frac{4-\tilde{\varepsilon}-z}{2} \\
\quad \text { (Anomalous) }\end{array}$ & $\begin{array}{l}\varrho_{3}(\delta)=\delta^{1-\varepsilon / 4} \\
\quad \text { (Anomalous) }\end{array}$ & $\begin{array}{c}\varrho_{4}(\delta)=\delta^{\frac{z}{2}\left(\frac{1}{(z-1)+\tilde{\varepsilon} / 2}\right)} \\
\text { (Anomalous) }\end{array}$ & $\begin{array}{l}\varrho_{5}(\delta)=\delta^{\frac{1}{1+\tilde{\varepsilon} / 2}} \\
\quad \text { (Anomalous) }\end{array}$ \\
\hline $\begin{array}{l}\text { Lagrangian } \\
\text { scaling law }\end{array}$ & $X^{2} \sim T$ & $X^{2} \sim T^{\frac{2}{4-\tilde{\varepsilon}-z}}$ & $X^{2} \sim T^{\frac{1}{1-\tilde{\varepsilon} / 4}}$ & $X^{2} \sim T^{+\frac{\tilde{\varepsilon}-2}{z}}$ & $X^{2} \sim T^{1+\tilde{\varepsilon} / 2}$ \\
\hline $\begin{array}{l}\text { Is effective } \\
\text { equation } \\
\text { local } \\
\text { diffusion } \\
\text { equation? }\end{array}$ & Yes & Yes & Yes & Yes & No \\
\hline $\begin{array}{l}\text { Is effective } \\
\text { equation } \\
\text { nonlocal } \\
\text { with } \\
\text { random } \\
\text { diffusivity? }\end{array}$ & No & No & No & No & Yes \\
\hline $\begin{array}{l}\text { Is effective } \\
\text { equation } \\
\text { dependent } \\
\text { on } v_{0} ?\end{array}$ & Yes & No & No & No & Yes \\
\hline $\begin{array}{l}\text { Is effective } \\
\text { diffusion } \\
\text { dependent } \\
\text { on ultraviolet } \\
\text { cut-off? }\end{array}$ & Yes & No & No & No & No \\
\hline $\begin{array}{l}\text { Is effective } \\
\text { diffusion } \\
\text { dependent } \\
\text { on infrared } \\
\text { cut-off? }\end{array}$ & No & Yes & Yes & No & No \\
\hline
\end{tabular}


and the bare diffusivity $v_{0}$, is completely irrelevant. Nevertheless, as indicated in Fig. 1 and Table 1, the exact renormalization for (1.4) with $z<2$ has very rich behavior with three distinct anomalous scaling regimes for $z$ with $1<z<2$ as $\tilde{\varepsilon}$ varies and an abrupt transition to two anomalous scaling regimes for $z$ with $0<z \leqq 1$. For $z>2$, there is strong enough temporal correlation at low wave numbers so that in hyperscaling regimes the renormalization theory coincides exactly with renormalization theory for the steady case discussed completely in Sect. 4. For the transition value, $z=2$, the scaling functions for the renormalization theory coincide at the same value of $\tilde{\varepsilon}$ with those for steady velocity fields from Sect. 4 but the formulas for the random diffusivity for $0<\tilde{e}<2$ are different and reflect the temporal statistics. The Eulerian scaling functions $\delta \mapsto \varrho(\delta)$, which gives rise to the non-trivial limiting equation for $\left\langle T^{\delta}\right\rangle$ can be directly related to the scaling law for the mean-square displacement of a particle undergoing the motion

$$
x(t)=\sqrt{v_{0}} \dot{B}_{1}(t), \quad \dot{y}(t)=v_{\delta}(x(t))+\sqrt{v_{0}} \dot{B}_{2}(t),
$$

where $B_{i}(t), i=1.2$ are independent Brownian motions. In fact, for particle displacements on the order of the ratio between the integral length scale and the dissipation length scale, the corresponding Lagrangian scaling law is,

$$
\frac{1}{T}=\varrho^{2}\left(\frac{1}{X}\right)
$$

Accordingly, for time-independent statistics, in the regime of mean-field theory, $\tilde{\varepsilon}<0$, we have $\varrho(\delta)=\delta$ and the mean-square particle displacements satisfy the scaling law

$$
X^{2} \sim T,
$$

while for $\tilde{\varepsilon}>2$, the anomalous Eulerian scaling function $\varrho(\delta)=\delta^{1-\tilde{\varepsilon} / 4}$ corresponds to

$$
X^{2} \sim T^{\frac{1}{1-\tilde{\varepsilon} / 4}} .
$$

In the intermediate region $0<\tilde{\varepsilon}<2$, where $\varrho(\delta)=\delta^{1 /(1+\tilde{\varepsilon} / 2)}$, we have the scaling

$$
X^{2} \sim T^{1+\tilde{\varepsilon} / 2} .
$$

The same type of results are valid in the time-dependent situation.

We conclude the introduction with several comments. The model problem in (1.4) with simple shearing has been used in mean field regimes quite often (see $[26,28])$ to illustrate the additional diffusivity in turbulent flows. In the case of steady velocities for (1.4), Matheron and de Marsily ([18]) have already observed anomalous scaling in their calculations of mean-square particle displacement but they did not attempt to derive effective equations for the first moment, $\bar{T}$. The examples with hyperscaling and renormalization from this paper provide examples for homogenization theory ([5]) with arbitrarily many length scales and without separation of scales. Of course, the model problem in (1.4) is the simplest special case of (1.1); nevertheless, the renormalization theory for this model is quite complex and provides some very interesting test problems for eddy viscosity theories for turbulence based on renormalization ideas. This aspect of the model problem is developed in another paper of the authors ([2]). 


\section{Velocity Statistics for the Model and Infrared Divergence for Turbulent Transport}

We begin this section by describing the problem of computing effective large scale transport equations for advection-diffusion by velocity fields with Kolmogoroff statistics in the inertial range. We show that this problem can be rescaled naturally to involve infrared divergence of the energy spectrum because there is no separation of scales and a continuous range of significantly excited modes between the largest scale motions and the smallest scale motions. We include this brief discussion because this reformulation with infrared divergence may not be known to the reader, although such divergence are implicit in some of the recent work on renormalization methods in turbulence $([8,17,27])$. This discussion also motivates the statistical structure for the velocity fields which we study in the model problem.

Kolmogoroff Statistics and Infrared Divergence. With $L_{0}$, the integral length scale and $\bar{U}$, the typical velocity of the energy containing but non-universal fluid motions, we introduce the Reynolds number, $\operatorname{Re}=\frac{\bar{U} L_{0}}{v}$, with $v$ the kinematic viscosity of the fluid. Our interest focuses on turbulent flows with $\operatorname{Re} \pi \infty$. The Kolmogorov hypothesis in $d$-space dimensions asserts (see [4]) that there is a dissipation length scale, $L_{d}$, so that as $\operatorname{Re} \rightarrow \infty$ the velocity energy spectrum has a universal form for wave numbers, $k$, in the range $L_{0}^{-1}<|k|<L_{d}^{-1}$ given by

$$
\left\langle|\hat{v}(k)|^{2}\right\rangle=C_{0} \bar{\varepsilon}^{2 / 3}|k|^{1-d-5 / 3} .
$$

Here $\wedge$ denotes Fourier transform, $\bar{\varepsilon}$ is the mean-dissipation rate, and $C_{0}$ is a universal constant. The energy spectrum is assumed to vanish for $|k|>L_{d}^{-1}$ or decay very rapidly while the velocity is not universal for variations on length scales larger than $L_{0}$. For simplicity in exposition, we have assumed that the velocity field in (2.1) is time-independent besides being incompressible. We consider the problem of advection diffusion of a quantity, $T$, by an incompressible steady velocity field with the Kolmogoroff velocity statistics from (2.1) and $\operatorname{div} v=0$, i.e.

$$
\frac{\partial T}{\partial t}+v \cdot \nabla T=\kappa \Delta T,\left.\quad T\right|_{t=0}=T_{0}(x) .
$$

Here $\kappa$ is the molecular diffusion coefficient. We assume that initially at time $t=0$, $T_{0}(x)$ varies substantially only on length scales larger than $L_{0}$; the objective of theories of "eddy-viscosity" modelling in this context is to determine an effective equation of motion involving variations of $T(x, t)$ on length scales larger than $L_{0}$ and for times at least comparable to the large scale eddy turnover time, $\bar{t}=L_{0} / \bar{U}$. We non-dimensionalize the equation in (2.2) by utilizing the dissipation length scale $L_{d}=\left(v^{3} / \vec{\varepsilon}\right)^{1 / 4}$ and dissipation time scale $t_{d}=(v / \bar{\varepsilon})^{1 / 2}$; first, using the relation $\bar{\varepsilon} \cong \frac{\bar{U}^{3}}{L_{3}}$, one finds that

$$
L_{d}=(\operatorname{Re})^{-3 / 4} L_{0}, \quad t_{d}=(\operatorname{Re})^{-1 / 2} \bar{t} .
$$

With this non-dimensionalization and the identification

$$
\delta=(\mathrm{Re})^{-3 / 4} \text {, }
$$


the equation in (2.2) assumes the form

$$
\frac{\partial T}{\partial t}+v_{\delta} \cdot \nabla T=\frac{1}{2} v_{0} \Delta T,\left.\quad T\right|_{t=0}=T_{0}(\delta x) .
$$

Here $\frac{1}{2} v_{0}=v / \kappa$ is the Prandtl number; for most fluids $v_{0}$ is quantity comparable to unity. The rescaled velocity field $v_{\delta}(x)$ has the energy spectrum

$$
\left\langle\left|\hat{v}_{d}(k)\right|^{2}\right\rangle= \begin{cases}C_{0}|k|^{1-d-5 / 3}, & \delta<|k|<1 \\ 0, & \text { otherwise }\end{cases}
$$

With the formulation in (2.4) and (2.5), the goal for "eddy viscosity" theories for high Reynolds number flows is to "homogenize" the problem in (2.4) and replace the dynamics in (2.4), (2.5) by an effective equation involving only the large scale motions in a fashion which is uniform as $\operatorname{Re} \rightarrow \infty$ or equivalently, as $\delta \rightarrow 0$. Furthermore for such an approximation to be useful, it must remain uniformly valid for time scales comparable to the eddy turnover time, $\bar{t}$; from (2.3) A) we see that the time for validity of these equations should be a long time at least comparable to $\delta^{-2 / 3}$ as $\delta \rightarrow 0$.

To continue the discussion and to point out some subtle aspects of this problem, we generalize the model problem in (2.4) and (2.5) by considering the parametrized family of incompressible velocity fields with energy spectrum given by

$$
\left\langle\left|\hat{v}_{\delta}(k)\right|^{2}\right\rangle= \begin{cases}C_{0}|k|^{2-d-\varepsilon}, & \delta<|k|<1 \\ 0, & \text { otherwise }\end{cases}
$$

where $\bar{\varepsilon},-\infty<\tilde{\varepsilon}<\infty$ is a parameter. The rescaled Kolmogoroff spectrum is given by $\bar{\varepsilon}=8 / 3$. The reader can regard $\tilde{\varepsilon}$ as the relevant expansion parameter in the $\tilde{\varepsilon}$-expansion of renormalization group theory $([17,8,27])$.

Basic work of Papanicoulaou, Varadhan and co-workers $([23,20])$ establishes that the standard formulas of homogenization theory yield an effective diffusivity for (2.4) provided that there is sufficient separation of scales between the velocity statistics and $T_{0}(\delta x)$. This work has been generalized under minimal hypotheses recently by the authors ([3]). Provided that the velocity spectrum satisfies

$$
\lim _{\delta \downarrow 0} \int_{R^{d}} \frac{\left\langle\left|\hat{v}_{\delta}(k)\right|^{2}\right\rangle}{|k|^{2}} d k<\infty,
$$

then the classical effective diffusion equation from homogenization theory remains valid with the expected long time diffusive scaling, $\delta^{2} t$. For the incompressible velocity field with spectrum given in (2.6) the condition in (2.7) is satisfied if and only if $\tilde{\varepsilon}<0$. The examples in this paper show that when the condition in (2.7) is violated, new effective equations with different anomalous shorter time scalings $\delta^{2 \theta} t, \theta<1$ arise; in some regimes these effective diffusion equations are non-local in space-time. For incompressible velocity fields with the Kolmogoroff spectrum in (2.5), we have $\tilde{\varepsilon}=8 / 3$, so there is infrared divergence as $\delta \downarrow 0$ and the integral in (2.7) diverges. In fact,

$$
\int_{R^{d}}\left\langle\left|\hat{v}_{\delta}(k)\right|^{2}\right\rangle d k \rightarrow \infty \quad \text { as } \quad \delta \rightarrow 0 \quad \text { for } \quad \tilde{\varepsilon}>2
$$


so there is even an infrared divergence of energy for $\tilde{\varepsilon}>2$. We remark that this fact is not inconsistent with the standard derivation of the Kolmogoroff spectrum which imposes finite energy at the outset. The choice of dissipation space and time scales from (2.3) A) and the fact that we require (2.4) to remain uniformly valid in the limit as $\operatorname{Re} \rtimes \infty$ results in the infrared divergence of the spectrum after this rescaling.

For a time dependent random incompressible velocity field, $v(x, t)$, the Kolmogoroff hypothesis yields the rescaled energy spectrum, ([4]),

$$
\left\langle\left|\hat{v}_{\delta}(k, \omega)\right|^{2}\right\rangle= \begin{cases}C_{0}|k|^{1-d-5 / 3}\left(|k|^{-2 / 3} \phi\left(\frac{\omega}{|k|^{2 / 3}}\right)\right), & \delta<|k|<1 \\ 0, & \text { otherwise }\end{cases}
$$

with $\wedge$ the space-time Fourier transform. Here $\phi \geqq 0$ is some structure function satisfying $\int \phi(s) d s=1$. The combination $\omega /|k|^{2 / 3}$ arises as the only combination of time and space frequencies independent of Re, consistent with the scaling in (2.3) A), and also with the energy spectrum, $E(k)=\int\left|\hat{v}_{\delta}(k, \omega)\right|^{2} d \omega$, given in (2.5) by the Kolmogoroff law although other space-time velocity statistics are possible. With this discussion, a natural generalization of the transport diffusion problem in (2.4), (2.5) to the time dependent case involves incompressible velocity fields $v_{\delta}(x, t)$ with

$$
\left\langle\left|\hat{v}_{\delta}(k, \omega)\right|^{2}\right\rangle= \begin{cases}C_{0}|k|^{2-d-\tilde{\varepsilon}}|k|^{-z} \phi\left(\frac{\omega}{|k|^{2}}\right), & \delta<|k|<1 \\ 0, & \text { otherwise }\end{cases}
$$

where $\tilde{\varepsilon}$ and $z$ with $0<z<\infty$ are parameters. From the preceding discussion, the Kolmogoroff spectrum corresponds to the values $\tilde{\varepsilon}=8 / 3$ and $z=2 / 3$ for $d=3$.

Velocity Statistics for the Model Problem. With the above discussion as motivation, we consider advection-diffusion for the model equation,

$$
\frac{\partial T}{\partial t}+v_{\delta}(x, t) \frac{\partial T}{\partial y}=\frac{1}{2} v_{0} \Delta T,\left.\quad T\right|_{t=0}=T_{0}(\delta x, \delta y) .
$$

First, we discuss the time-independent case. With (2.6) for $d=1$ as motivation, we assume that the steady velocity field $v_{\delta}^{s}(x)$ has stationary Gaussian statistics with the spectral representation

$$
v_{\delta}^{s}(x)=(2 \pi)^{-1 / 2} \int e^{i k z}|k|^{\frac{1-\tilde{\varepsilon}}{2}} \psi_{0}^{1 / 2}\left(\frac{|k|}{\delta}\right) \psi_{\infty}^{1 / 2}(|k|) W(d k),
$$

where $W(d k)$ is Gaussian white noise satisfying

$$
\left\langle W(d k) \overline{W\left(d k^{\prime}\right)}\right\rangle=\delta\left(k+k^{\prime}\right) d k .
$$

The function $\psi_{\infty}^{1 / 2}(k)$ is the ultraviolet cut-off and is a rapdily decreasing function with

$$
\begin{gathered}
\psi_{\infty}^{1 / 2} \geqq 0, \quad \psi_{\infty}^{1 / 2} \text { continuous at zero, and } \psi_{\infty}^{1 / 2}(0)=1 \\
\int|l|^{\alpha} \psi_{\infty}(k) d k \leqq C_{\alpha}, \quad \alpha>0 .
\end{gathered}
$$


The function $\psi_{0}^{1 / 2}(|k| / \delta)$ is the infrared cut-off and satisfies $\psi_{0}^{1 / 2}(k) \geqq 0$ and also, for some constants $k_{1}, k_{0}$ with $k_{1}>k_{0}$,

$$
\psi_{0}(|k|) \equiv 1 \quad \text { for }|k|>k_{1}, \quad \psi_{0}(|k|) \equiv 0 \text { for }|k|<k_{0} .
$$

The velocity field $v_{\delta}^{s}(x)$ in (2.11) has the energy spectrum

$$
\left\langle\left|\hat{v}_{\delta}^{s}(k)\right|^{2}\right\rangle=(2 \pi)^{-1 / 2}|k|^{1-\tilde{\varepsilon}} \psi_{o}\left(\frac{|k|}{\delta}\right) \psi_{\infty}(|k|)
$$
with the same general form as that in (2.6) with $d=1$. In (2.6) $\psi_{0}\left(\frac{|k|}{\delta}\right)$ and $\psi_{\infty}(|k|)$
correspond to the characteristic functions of $|k|>\delta$ and $|k|<1$.

For the time dependent velocity fields, $v_{\delta}(x, t)$, in (2.10) we assume stationary Gaussian statistics with our motivation from (2.9) with $d=1$. We assume that $v_{\delta}(x, t)$ has Gaussian statistics with the spectral $\delta$ representation

$$
\begin{aligned}
v_{\delta}(x, t)= & (2 \pi)^{-1 / 2} \iint e^{i(x k+\omega t)} \psi_{0}^{1 / 2}\left(\frac{|k|}{\delta}\right) \psi_{\infty}^{1 / 2}(|k|)|k|^{\frac{1-\tilde{\varepsilon}}{2}} \\
& \pi^{-1 / 2} a^{1 / 2}|k|^{z / 2}\left(i \omega+a|k|^{z}\right)^{-1} W(d k) \tilde{W}(d \omega)
\end{aligned}
$$

with $W(d k)$ and $\tilde{W}(d \omega)$ independent Gaussian white noise measures. Note that they satisfy

$$
\begin{aligned}
\left\langle W(d k) W(d \omega) \overline{W\left(d k^{\prime}\right)} \overline{W\left(d \omega^{\prime}\right)}\right\rangle & =\left\langle W(d k) \overline{W\left(d k^{\prime}\right)}\right\rangle\left\langle W(d \omega) \overline{W\left(d \omega^{\prime}\right)}\right\rangle \\
& =\delta\left(k+k^{\prime}\right) \delta\left(\omega+\omega^{\prime}\right) d k d \omega .
\end{aligned}
$$

The energy-power spectrum of the unsteady velocity field in (2.15) is given by

$$
\left\langle\left|\tilde{v}_{\delta}(k, \omega)\right|^{2}\right\rangle=\psi_{0}\left(\frac{|k|}{\delta}\right) \psi_{\infty}(|k|)|k|^{1-\tilde{\varepsilon}}\left(\pi^{-1} a|k|^{z}\left(\omega^{2}+a^{2}|k|^{2 z}\right)^{-1}\right) .
$$

With the energy spectrum for the steady case from (2.14), we see that the energypower spectrum for $\hat{v}_{\delta}(k, \omega)$ has the form

$$
\left\langle\left|\hat{v}_{\delta}(k, \omega)\right|^{2}\right\rangle=\left|\hat{v}_{\delta}^{s}(k)\right|^{2}(a|k|)^{-z} \phi\left(\frac{\omega}{a|k|^{2}}\right),
$$

where $\phi(s)$ is the time frequency structure function,

$$
\phi(s)=\pi^{-1}\left(1+s^{2}\right)^{-1} .
$$

We make the same assumptions on $\psi_{0}$ and $\psi_{\infty}$ as we used earlier in the steady case and impose the restriction, $0<z<\infty$ on the fixed parameter $z$. Looking back at the formulae in (2.8) and (2.9), we observe that the velocity spectrum for the model problem as given in (2.16), (2.17) has the same form as the general time-dependent case described earlier; the parameter values $\tilde{\varepsilon}=8 / 3$ and $z=2 / 3$ in the model problem correspond to the Kolmogoroff spectrum. Of course, turbulent velocity fields obey only approximate Gaussian statistics and this is one simplification for the model problem which we use throughout this paper. 


\section{Representation Formulas and Identities for Renormalization for the Model Problem}

Here we develop representation formulas for the solution of the model advectiondiffusion equation

$$
\frac{\partial T}{\partial t}+v_{\delta}(x, t) \frac{\partial T}{\partial y}=\frac{1}{2} v_{0} \Delta T,\left.\quad T\right|_{t=0}=T_{0}(\delta x, \delta y),
$$

where the velocity $v_{\delta}(x, t)$ has the stationary Gaussian statistics described in (2.11) for the steady case and in (2.15) for the general dependent case.

First, we use Fourier analysis and the Feynman-Kac formula to develop a representation for the solution of (3.1) for each fixed realization of the velocity field, $v_{\delta}(x, t)$. The initial function $T_{0}(\delta x, \delta y)$ is given by the Fourier integral

$$
T_{0}(\delta x, \delta y)=(2 \pi)^{-1} \iint e^{i(\delta x \eta+\delta y \xi)} \widehat{T}_{0}(\eta, \xi) d \xi d \eta,
$$

thus, we only need to find a representation for the problem in (3.1) with initial data $e^{i(\delta x \eta+\delta y \xi)}$. With $\eta^{\prime}=\delta \eta, \xi^{\prime}=\delta \xi$, we seek the solution of (3.1) with this initial data in the form,

$$
e^{i\left(y \xi^{\prime}\right)} \hat{p}\left(x, t, \xi^{\prime}, \eta^{\prime}\right),
$$

where $\hat{p}$ satisfies the equation

$$
\frac{\partial \hat{p}}{\partial t}+i v_{\delta}(x, t) \xi^{\prime} \hat{p}=-\frac{v_{0}}{2} \xi^{\prime 2} \hat{p}+\frac{v_{0}}{2} \hat{p}_{x x},\left.\quad \hat{p}\right|_{t=0}=e^{i \eta^{\prime} x} .
$$

We represent the solution of (3.3) for each fixed realization of the velocity, $v_{\delta}(x, t)$ by the Feynman-Kac formula ([19]),

$$
\begin{aligned}
\hat{p}\left(x, t, \xi^{\prime} \eta^{\prime}\right)= & e^{i \eta^{\prime} x} e^{-\frac{v_{0}}{2}\left(\xi^{\prime}\right) 2 t} \\
& \times E\left[e^{i \eta^{\prime} v_{0}^{\prime 2} / 2 \beta(e)} e^{i \xi^{\prime} v_{\delta}^{t} v_{\delta}\left(x+v_{0}^{1 / 2} \beta(s), t-s\right) d s}\right] .
\end{aligned}
$$

Here and in the remainder of this paper $E[\cdot]$ denotes the integral over Weiner measure in the space of continuous functions. With the identification, $\xi^{\prime}=\delta \xi$, $\eta^{\prime}=\delta \eta$, and (3.2)-(3.4), we have the solution of (3.1) for each fixed realization of $v_{\delta}$ as the function space integral,

$$
\begin{aligned}
T(x, y, t)= & (2 \pi)^{-1} \iint e^{i \delta x \eta+\delta y \xi} e^{-\frac{v_{0}}{2} \delta^{2}(\xi)^{2 t}} \widehat{T}_{0}(\eta, \xi) \\
& \times E\left[e^{\left.i \delta \eta \eta_{0}^{1 / 2 \beta(t)}\right)} e^{-i \delta \xi \xi^{t} v_{d}\left(x+v_{0}^{1 / 2} \beta(s), t-s\right)}\right] d \xi d \eta .
\end{aligned}
$$

The Representation Formula. Formula (3.5) gives the solution of the initial-value problem for every realization of the random velocity field $v_{\delta}$; i.e. (3.5) is not yet averaged over the velocity statistics given by the models (2.11) and (2.15) in the steady and time-dependent cases, respectively. Our objective here is to find an effective equation describing the large scale long-time dynamics of (3.1); thus, we seek suitable space-time rescalings

$$
x^{\prime}=\delta x, \quad y^{\prime}=\delta y, \quad t^{\prime}=\varrho^{2}(\delta) t
$$


with $\varrho^{2}(\delta) \rightarrow 0$ as $\delta \rightarrow 0$. The scaling function $\varrho^{2}(\delta)$ will be determined for fixed values of $\tilde{\varepsilon}$ and $z$ by the nature of the statistics in (2.11) and (2.15). (Classical diffusive scaling occurs when $\varrho(\delta)=\delta$.) With this suitable choice of scaling functions, our next goal is to compute an effective representation for

$$
\bar{T}\left(x^{\prime}, y^{\prime}, t^{\prime}\right)=\lim _{\delta \downarrow 0}\left\langle T\left(\frac{x^{\prime}}{\delta}, \frac{y^{\prime}}{\delta}, \frac{t^{\prime}}{\varrho^{2}(\delta)}\right)\right\rangle .
$$

The equation satisfied by $\bar{T}\left(x^{\prime}, y^{\prime}, t^{\prime}\right)$ is the effective "turbulent viscosity" equation. Here and below, $\langle\cdot\rangle$ denotes ensemble average over velocity statistics. With (3.5)-(3.7) we have

$$
\begin{aligned}
\left\langle T\left(\frac{x^{\prime}}{\delta}, \frac{y^{\prime}}{\delta}, \frac{t^{\prime}}{\varrho^{2}(\delta)}\right)\right\rangle= & (2 \pi)^{-1} \iint e^{-\left(x^{\prime} \eta+y^{\prime} \xi\right)} e^{-\frac{v_{0} \delta^{2}}{2} \varrho^{2} \xi^{2 t^{\prime}}} \hat{T}_{0}(\xi, \eta) \\
& \times\left[e^{i \delta \eta v_{0}^{1 / 2} \beta \frac{t^{\prime}}{\varrho^{2}}}\left\langle e^{-i \delta \xi^{t^{\prime} / \rho^{2}} v_{\delta}^{2}\left(\frac{x^{\prime}}{\delta}+v_{0}^{\prime 2} \beta(s), \frac{t^{\prime}}{\varrho^{2}}-s\right) d s}\right\rangle\right] d \xi d \eta .
\end{aligned}
$$

To simplify the expression in (3.8) further, we use the well-known fact that Brownian motion rescales ([19]) so that

$$
\beta\left(t^{\prime} / \varrho^{2}\right) \cong \frac{1}{\varrho} \beta\left(t^{\prime}\right) \text { in law }
$$

for any positive number $\varrho$. We also make crucial use of the assumption of Gaussian statistics given in (2.11) and (2.15). We recall the following general fact ([9]):

If $u(x, t)$ is a mean-zero stationary Gaussian process, and $\beta(s)$ is a fixed continuous function, then

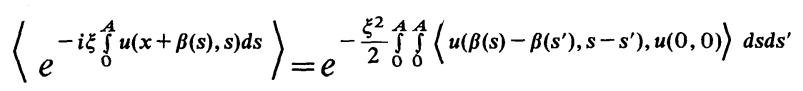

With the stationary Gaussian statistics from (2.11) and (2.15) and (3.10), we obtain for fixed $\beta$ that

$$
\begin{aligned}
& \left\langle e^{-i \delta \xi^{t^{\prime \prime} / \rho^{2}} v_{\delta}\left(\frac{x^{\prime}}{\delta}+v_{0}^{1 / 2} \beta(s) \cdot \frac{t^{\prime}}{\varrho^{2}}-s\right) d s}\right\rangle \\
& =e^{-\frac{\delta^{2} \xi^{2}}{2} t^{\prime} / \rho^{2} t^{\prime} / \rho^{2}\left\langle v_{\delta}\left(v_{0}^{1 / 2}\left(\beta(s)-\beta\left(s^{\prime}\right)\right), s-s^{\prime}\right) v_{\delta}(0,0)\right\rangle d d d s^{\prime}} \\
& =e^{-\frac{\xi^{2}}{2} \frac{\delta^{2} t^{\prime 2}}{\varrho^{4}} \int_{0}^{1} \int_{0}^{1}\left\langle v_{\delta}\left(\frac{\left(v_{0} t^{1}\right)^{1 / 2}}{\varrho}\left(\beta(s)-\beta\left(s^{\prime}\right)\right), \frac{t^{\prime}}{\varrho^{2}}\left(s-s^{\prime}\right)\right) v_{\delta}(0,0)\right\rangle d s d s^{\prime}} .
\end{aligned}
$$

In the last identity from (3.11), we applied the fact in (3.9) after changing variables. The double integral appearing in the last exponent in (3.11) can be written explicitly in terms of the velocity statistics; from (2.11) and (2.15) we have the identity,

$$
\left\langle v_{\delta}(\hat{x}, \hat{t}) v_{\delta}(0,0)\right\rangle \equiv R_{\delta}(\hat{x}, \hat{t})=(2 \pi)^{-1} \int e^{i \hat{x} k} e^{-a|k| z|\hat{t}|} \psi_{0}\left(\frac{|k|}{\delta}\right) \psi_{\infty}(|k|)|k|^{1-\tilde{\varepsilon}} d k
$$

(the formula for the steady case is (3.12) with $a=0$ as the reader can easily verify). 
With the formulas in (3.8)-(3.12), we obtain the Representation Formula,

$$
\begin{aligned}
& \bar{T}\left(x^{\prime}, y^{\prime}, t^{\prime}\right)=\left\langle T\left(\frac{x^{\prime}}{\delta}, \frac{y^{\prime}}{\delta}, \frac{t^{\prime}}{\varrho^{2}(s)}\right)\right\rangle=(2 \pi)^{-1} \iint e^{i\left(x^{\prime} \eta+y^{\prime} \xi\right)} e^{-\frac{v_{0}}{2} \frac{\delta^{2}}{\varrho^{2} t^{\prime}}} \widehat{T}_{0}(\xi, \eta) \\
& \quad \times E\left[e^{i \frac{\delta}{\varrho} \eta\left(v_{0} t^{\prime}\right)^{1 / 2} \beta(1)} e^{-\frac{\xi^{2}}{2} \frac{\delta^{2} t^{\prime 2}}{\varrho^{4}} \int_{0}^{1} \int_{0}^{1} R_{\delta}\left(\frac{\left(\nu_{0} t^{\prime}\right)^{1 / 2}}{\varrho}\left(\beta(s)-\beta\left(s^{\prime}\right)\right), \frac{t^{\prime}}{\varrho^{2}}\left(s-s^{\prime}\right)\right) d s d s^{\prime}}\right] d \xi d \eta .
\end{aligned}
$$

For the remainder of this paper including Sects. 4 and 5, for notational convenience, we will drop the ' as superscripts in most identities referring to the formula in (3.13).

Identities for Renormalization. As we have mentioned earlier, whenever $\varrho=\delta$ we have standard diffusive scaling and mean field theory occurs. On the other hand, in Sects. 4 and 5 we show that when renormalization occurs, there is a scaling law $\varrho(\delta)$ satisfying $\delta / \varrho(\delta) \downarrow 0$ as $\delta \rightarrow 0$. In this regime we have

$$
\begin{gathered}
\frac{\delta}{\varrho} \eta v_{0}^{1 / 2} \beta(t) \rightarrow 0, \\
e^{-\frac{v_{0}}{2} \frac{\delta^{2}}{\varrho^{2}} \xi^{2} t} \rightarrow 1 \quad \text { provided } \delta / \varrho \rightarrow 0 .
\end{gathered}
$$

Thus, with $Y_{\delta}(\beta, t)$ given by

$$
Y_{\delta}(\beta, t)=\frac{\delta^{2} t^{2}}{2 \varrho^{4}} \int_{0}^{1} \int_{0}^{1} R_{\delta}\left(\frac{\left(v_{0} t\right)^{1 / 2}}{\varrho}\left(\beta(s)-\beta\left(s^{\prime}\right)\right), \frac{t}{\varrho^{2}}\left(s-s^{\prime}\right)\right) d s d s^{\prime},
$$

our strategy in Sects. 4 and 5 below will always involve finding functions of $\beta$, $Y(\beta, t), \widetilde{Y}(\beta, t)$, and a suitable scaling law with $\delta / \varrho \rightarrow 0$ so that

$$
\begin{aligned}
& \text { A) }\left|Y_{\delta}(\beta, t)\right| \leqq \tilde{Y}(\beta, t), \\
& \text { B) } E[\tilde{y}(\beta, t)]<\infty, \\
& \text { C) } \lim _{\delta \rightarrow 0} Y_{\delta}(\beta, t) \rightarrow Y(\beta, t) \neq 0 \text {, pointwise in } \beta .
\end{aligned}
$$

With (3.14)-(3.16), it follows from applying the dominated convergence theorem to (3.13) that with a suitable choice of the scaling function $\varrho(\delta)$ with $\delta / \varrho(\delta) \downarrow 0$, the solution for the effective equation is given by

$$
\bar{T}(x, y, t)=(2 \pi)^{-1} \iint e^{i(x \eta+y \xi)} E\left[e^{-\xi^{2} Y(\beta, t)}\right] \widehat{T}_{0}(\xi, \eta) d \xi d \eta .
$$

An appropriate way to guess the scaling function $\varrho(\delta)$ is to require $E\left(Y_{\delta}(\beta, t)\right)$ $\leqq C_{0}<\infty$ as $\delta \downarrow 0$. To achieve the behavior in (3.16), (3.17) in the hyperscaling regime where $\delta / \varrho(\delta) \downarrow 0$, it will be useful to substitute the identity in (3.12) into the definition for $Y_{\delta}(\beta, t)$ in (3.15) and then to rescale the frequency variable by the scaling function, $k^{\prime}=k / g(\delta)$. The result is the identity

$$
\begin{aligned}
Y_{\delta}(\beta, t)= & \frac{g(\delta)^{2-\varepsilon} \delta^{2}}{\varrho^{4}} \frac{t^{2}}{2}(2 \pi)^{-1} \int_{0}^{1} \int_{0}^{1} \int e^{i \frac{g(\delta)}{\varrho}\left(v_{0} t\right)^{1 / 2}\left(\beta(s)-\beta\left(s^{\prime}\right)\right) k} e^{-\frac{a t}{\varrho^{2}(g(\delta)) z|k|^{z}\left|s-s^{\prime}\right|}} \\
& \times \psi_{0}\left(\frac{g(\delta)}{\delta}|k|\right) \psi_{\infty}(g(\delta) k)|k|^{1-\tilde{\varepsilon}}|k|^{1-\tilde{\varepsilon}} d k d s d s^{\prime}
\end{aligned}
$$


We will make a variety of choices of $\varrho(\delta)$ and $g(\delta)$ in the renormalization calculations in Sects. 4 and 5 and the following comments provide useful guidelines:
A) If $g(\delta) \downarrow 0$ as $\delta \downarrow 0$, as a consequence of (2.12) B), $\psi_{\infty}(g(\delta) k) \rightarrow 1$ and the ultraviolet cut-off is negligible in the limit.
B) If $g(\delta) / \delta \succ \infty$ as $\delta \downarrow 0$, the infrared cut-off is negligible because
$\psi_{0}\left(\frac{g(\delta)}{\delta}|k|\right) \rightarrow 1$ as $\delta \downarrow 0$.
C) If $g(\delta) / \varrho(\delta) \downarrow 0$ as $\delta \downarrow 0$, the effects of Brownian motion are negligible in the limit for $Y_{\delta}(\beta, t)$.

Of course all of the comments in (3.19) are true only under the tacit assumption that the integrand in (3.18) is uniformly integrable as $\delta \downarrow 0$ so that the dominated convergence theorem applies.

\section{Renormalization with Steady Velocity Fields}

Here we study renormalization for the model problem,

$$
\frac{\partial T}{\partial t}+v_{\delta}(x) \frac{\partial T}{\partial y}=\frac{1}{2} v_{0} \Delta T,\left.\quad T\right|_{t=0}=T_{0}(\delta x, \delta y),
$$

where $v_{\delta}(x)$ has the stationary Gaussian statistics in (2.11). For the problem in (4.1), the representation formula in (3.13) for the averaged solution becomes

$$
\begin{aligned}
\left\langle T\left(\frac{x}{\delta}, \frac{y}{\delta}, \frac{t}{\varrho^{2}(\delta)}\right)\right\rangle= & (2 \pi)^{-1} \iint e^{i(x \eta+y \xi)} e^{-\frac{v_{0}}{2} \frac{\delta^{2}}{\varrho^{2} \xi^{2} t}} \widehat{T}_{0}(\xi, \eta) \\
& \times E\left[e^{i \frac{\delta}{2} \eta\left(v_{0} t\right)^{1 / 2} \beta(1)} e^{-\xi^{2} Y_{\delta}(\beta, t)}\right] d \xi d \eta
\end{aligned}
$$

with

and

$$
\begin{aligned}
Y_{\delta}(\beta, t)= & \frac{\delta^{2}}{\varrho^{4}} \frac{t^{2}}{2} \int_{0}^{1} \int_{0}^{1} R_{\delta}\left(\frac{\left(v_{0} t\right)^{1 / 2}}{\varrho}\left(\beta(s)-\beta\left(s^{\prime}\right)\right)\right) d s^{\prime} d s \\
= & \frac{\delta^{2}}{\varrho^{4}} \frac{t^{2}}{2}(2 \pi)^{-1} \int_{0}^{1} \int_{0}^{1} \int e^{i \frac{\left(v_{0} t\right)^{1 / 2}}{\varrho}\left(\beta(s)-\beta\left(s^{\prime}\right)\right) k} \\
& \times \psi_{0}\left(\frac{|k|}{\delta}\right) \psi_{\infty}(|k|)|k|^{1-\tilde{\varepsilon}} d k d s^{\prime} d s
\end{aligned}
$$

$$
R_{\delta}(\hat{x})=(2 \pi)^{-1} \int e^{i \hat{x} k} \psi_{0}\left(\frac{|k|}{\delta}\right) \psi_{\infty}(|k|)|k|^{1-\tilde{\varepsilon}} d k
$$

As mentioned in the introduction, here we prove that there are three different scaling regimes with completely different behavior for this problem as $\tilde{\varepsilon}$ varies: the regime with $\tilde{\varepsilon}<0$ where mean-field theory applies; the anomalous scaling regime 
with $2<\tilde{\varepsilon}<4$, where the bare viscosity $v_{0}$ is irrelevant and inviscid dynamics dominates; the anomalous scaling regime $0<\tilde{\varepsilon}<2$, where the effective diffusion equation is non-local with a random diffusivity given by a rich variety of explicit formulas which depend on the bare diffusivity, $v_{0}$. We systematically apply the representation formula in (4.2) and all of the systematic ideas for renormalization described in (3.12)-(3.19). After a brief discussion of the other regimes in $\tilde{\varepsilon}$, we mostly emphasize the rather subtle behavior that occurs for $0<\tilde{\varepsilon}<2$.

A) The Regime $\tilde{\varepsilon}<0-$ Mean Field Theory. The regime with $\tilde{\varepsilon}<0$ is precisely the region where the limit as $\delta \downarrow 0$ of the integral in (2.7) is finite for the steady velocity statistics from (2.11) that we assume in this section. Thus, from the general theory of homogenization described briefly above $(2.7)$ (see $[23,20,3]$ ), it follows that the conventional diffusive scaling, $\varrho(\delta)=\delta$, is appropriate and the effective averaged equation valid at large scales and long times for (4.1) for $\tilde{\varepsilon}<0$ is given by

$$
\frac{\partial \bar{T}}{\partial t}=\frac{1}{2} v_{0} \Delta \bar{T}+v_{0}^{-1} D(\tilde{\varepsilon}) \bar{T}_{y y},\left.\quad \bar{T}\right|_{t=0}=T_{0}(x, y),
$$

where

$$
D(\tilde{\varepsilon})=(\pi)^{-1} \int|k|^{-1-\tilde{\varepsilon}} \psi_{\infty}(|k|) d k .
$$

We observe that the integral in (4.5) converges for small wave numbers only when $\tilde{\varepsilon}$ satisfies $\tilde{\varepsilon}<0$. A simple self-contained proof of the mean field theory results in (4.4), (4.5) which utilizes the explicit representation in (4.2) is presented in the appendix to this paper.

B) The Regime $2 \leqq \tilde{\varepsilon}<4$ - the Inviscid Hyperscaling Region. The integral

$$
\int \psi_{0}(|k|)|k|^{1-\tilde{\varepsilon}} d k
$$

is convergent for $\tilde{\varepsilon}>2$. This fact suggests the frequency scaling factor $g(\delta)=\delta$ in (3.18). With this choice,

$$
\begin{aligned}
Y_{\delta}(\beta, t)= & \frac{\delta^{4-\varepsilon}}{\varrho^{4}} \frac{t^{2}}{2}(2 \pi)^{-1} \int_{0}^{1} \int_{0}^{1} \int e^{i \frac{\delta}{\varrho}\left(v_{0} t\right)^{1 / 2}\left(\beta(s)-\beta\left(s^{\prime}\right)\right) k} \\
& \times \psi_{0}(|k|) \psi_{\infty}(\delta|k|)|k|^{1-\varepsilon} d k d s^{\prime} d s .
\end{aligned}
$$

The facts in (4.5) and (4.6) suggest the scaling function $\varrho(\delta)$ so that $\delta^{4-\tilde{\varepsilon}}=\varrho^{4}$, i.e.

$$
\varrho(\delta)=\delta^{1-\tilde{\varepsilon} / 4}, \quad 2<\tilde{\varepsilon}<4 .
$$

We require that $\tilde{\varepsilon}$ satisfies $\tilde{\varepsilon}<4$ so that $\varrho(\delta) \rightarrow 0$ as $\delta \downarrow 0$. Since $\delta / \varrho(\delta) \downarrow 0$, the contribution from the Brownian motion is negligible in the limit as $\delta \downarrow 0$ and

$$
\lim _{\delta \rightarrow 0} Y_{\delta}(\beta, t)=\frac{t^{2}}{2}(2 \pi)^{-1} \int \psi_{0}(|k|)|k|^{1-\tilde{\varepsilon}} d k .
$$

Thus, from (3.14)-(3.20), the dominated convergence theorem, and (4.8), we conclude that the effective averaged diffusion equation at the large spatial scales with the long-time scale defined by $\varrho(\delta)=\delta^{1-\tilde{\varepsilon} / 4}$ is given by

$$
\frac{\partial \bar{T}}{\partial t}=t D(\tilde{\varepsilon}) \bar{T}_{y y},\left.\quad \bar{T}\right|_{t=0}=T_{0}(x, y)
$$


with the effective diffusion coefficient given by

$$
D(\tilde{\varepsilon})=(2 \pi)^{-1} \int \psi_{0}(|k|)|k|^{1-\tilde{\varepsilon}} d k,
$$

for $\tilde{\varepsilon}>2$. Suppose now that $\tilde{\varepsilon}=2$. Then the integral in (4.6) diverges logarithmically as $\delta \rightarrow 0$. We see from (4.6) with $\tilde{\varepsilon}=2$ that the correct choice of scaling function $\varrho(\delta)$ must be such that

$$
\frac{\delta^{2}}{\varrho^{4}} \log \frac{1}{\delta}=1, \quad \text { or } \quad \varrho(\delta)=\left(\log \frac{1}{\delta}\right)^{1 / 4} \delta^{1 / 2} .
$$

With this choice of scaling function we have, again from (4.6),

$$
\lim _{\delta \downarrow 0} Y_{\delta}(\beta, t)=\frac{t^{2}}{4 \pi} .
$$

Thus, the effective equation for $\bar{T}$ when $\tilde{\varepsilon}=2$ is

$$
\frac{\partial \bar{T}}{\partial t}=\frac{t}{4 \pi} \bar{T}_{y y} .
$$

We remark that the scaling function $\varrho(\delta)$ satisfies $\varrho(\delta)=\delta^{1 / 2}$ up to logarithmic factors at $\tilde{\varepsilon}=2$. This value corresponds to the natural convective scaling, $T\left(\frac{x}{\delta}, \frac{y}{\delta}, \frac{t}{\delta}\right)$, associated with (4.1) with $v_{0}=0$. For $\tilde{\varepsilon}>2$, the scale $\varrho(\delta)=\delta^{1-\tilde{\varepsilon} / 4}$ defines a shorter time scale than the natural inviscid time scale - a regime of hyperscaling dominated by inviscid transport. To emphasize this point, we briefly consider the problem of determining the large spatial scale and long-time averaged equation for the inviscid problem

$$
\frac{\partial T}{\partial t}+v_{\delta}(x) \frac{\partial T}{\partial y}=0,\left.\quad T\right|_{t=0}=T_{0}(\delta x, \delta y)
$$

with the same velocity statistics from (2.11) as we used in discussing (4.1). The representation formula from (4.2) applies to the rescaled solution by setting $v_{0} \equiv 0$ with $R_{\delta}$ given in (4.3) A). For the finite energy regime, $\tilde{\varepsilon}<2$, with

$$
\int|k|^{1-\tilde{\varepsilon}} \psi_{\infty}(|k|) d k<\infty,
$$

it follows from (3.13) that the appropriate time scaling for (4.11) is given by the standard convective scale

$$
\varrho=\delta^{1 / 2}, \text { for } \tilde{\varepsilon}<2
$$

with corresponding averaged equation for the inviscid problem given by

$$
\frac{\partial \bar{T}}{\partial t}=t D_{0}(\tilde{\varepsilon}) \bar{T}_{y y}, \quad D_{0}(\tilde{\varepsilon})=(2 \pi)^{-1} \int|k|^{1-\tilde{\varepsilon}} \psi_{\infty}(|k|) d k .
$$

On the other hand, for $\tilde{\varepsilon} \geqq 2$, the regime with infinite kinetic energy, hyperscaling occurs for the problem in (4.11) with the same scaling law and effective equation from (4.7), (4.9), and (4.10) as occurs in the renormalization of (4.1) for $\tilde{\varepsilon} \geqq 2$. This justifies our terminology for calling the regime with $\tilde{\varepsilon} \geqq 2$, the region of hyperscaling for (4.1) dominated by renormalization for the inviscid problem. 
C) The Regime $0<\tilde{\varepsilon}<2-$ The Region with Random Nonlocal Diffusivity. As in the case just described, we seek a frequency scaling factor $k^{\prime}=g(\delta) k$ which renormalizes $Y_{\delta}(\beta, t)$ defined in (4.3). The simple choices $g(\delta)=1$ and $g(\delta)=\delta$ both fail for $0<\tilde{\varepsilon}<2$ because both

$$
\int|k|^{1-\tilde{\varepsilon}} \psi_{0}(k) d k \text { and } \int|k|^{-1-\tilde{\varepsilon}} \psi_{\infty}(k) d k
$$

are divergent integrals. Instead for fixed $t$, we make the intermediate choice, $g(\delta)$ $=\varrho(\delta)\left(v_{0} t\right)^{-1 / 2}$ and anticipate that $\varrho(\delta)$ is a hyperdiffusive scaling so that $\delta / \varrho(\delta) \rightarrow 0$; with this choice of $g(\delta)$ in (3.18), the path integral $Y_{\delta}(\beta, t)$ from (4.3) A) has the form,

$$
\begin{aligned}
Y_{\delta}(\beta, t)= & \frac{\delta^{2}}{\varrho^{2+\tilde{\varepsilon}}}\left(v_{0}\right)^{\varepsilon / 2-1} \frac{t^{1+\tilde{\varepsilon} / 2}}{2}(2 \pi)^{\frac{-1}{2}}\left(\int\left((2 \pi)^{-1 / 2} \int_{0}^{1} \int_{0}^{1} e^{i\left(\beta(s)-\beta\left(s^{\prime}\right)\right) k} d s d s^{\prime}\right)\right. \\
& \left.\times\left(\psi_{0}\left(\frac{\varrho\left(v_{0} t\right)^{-1 / 2}}{\delta}|k|\right) \psi_{\infty}\left(\varrho\left(v_{0} t\right)^{-1 / 2}|k|\right)|k|^{1-\tilde{\varepsilon}} d k\right)\right) .
\end{aligned}
$$

We pick the time rescaling $\varrho(\delta)$ so that $\delta^{2}=\varrho^{2+\tilde{\varepsilon}}$ and anticipate that the integral on the right-hand side of (4.12) has a finite limit as $\delta \downarrow 0$. Thus, the scaling law is given by

$$
\varrho(\delta)=\delta^{\frac{1}{1+\tilde{\varepsilon} / 2}} \text { for } 0<\tilde{\varepsilon}<2 .
$$

We remark that $\varrho(\delta)=\delta$ at $\tilde{\varepsilon}=0$ while $\varrho(\delta)=\delta^{1 / 2}$ at $\tilde{\varepsilon}=2$ so the scalings in the regime $0<\tilde{\varepsilon}<2$ are diffusive/convective and intermediate between purely diffusive scaling and inviscid convective scaling.

We concentrate on investigating the limiting behavior for the integral in parentheses on the right-hand side of (4.12). We define

$$
\widehat{F}_{\delta}^{\check{\varepsilon}}(k)=\psi_{0}\left(\frac{\varrho}{\delta}\left(v_{0} t\right)^{-1 / 2}|k|\right) \psi_{\infty}\left(\varrho\left(v_{0} t\right)^{-1 / 2}|k|\right)|k|^{1-\tilde{\varepsilon}},
$$

and remark that as $\delta \downarrow 0$,

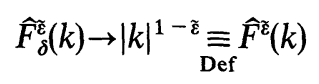

pointwise. The continuous map from the unit square to the real line given by $\left(s, s^{\prime}\right) \mapsto \beta\left(s^{\prime}\right)-\beta(s)$ pushes forward Lebesgue measure on the square to a finite Borel measure, $\mu_{\beta}$, on the real line via the formula

$$
\int g(x) d \mu_{\beta} \equiv \int_{\text {Def }}^{1} \int_{0}^{1} g\left(\beta(s)-\beta\left(s^{\prime}\right)\right) d s^{\prime} d s
$$

for every continuous function $g(x)$ which vanishes at infinity. The function $\widehat{F}_{\delta}^{\tilde{\varepsilon}}(k)$ belongs to $L^{1} \cap L^{\infty}$ for fixed $\delta$ and therefore has an inverse Fourier transform $F_{\delta}^{\widetilde{\varepsilon}}(x)$ $=(2 \pi)^{-1 / 2} \int e^{i x \cdot k} \hat{F}_{\delta}^{\tilde{\varepsilon}}(k) d k$ which is a continuous function vanishing at infinity. By Plancherel's theorem with (4.14) and (4.15) the integral on the right-hand side of (4.12) is given by

$$
\int \widehat{\mu}_{\beta}^{(k)} \hat{\mathrm{F}}_{\delta}^{\tilde{\varepsilon}}(k) d k=\int F_{\delta}^{\tilde{\varepsilon}}(x) d \mu_{\beta} .
$$

Below, we will show that as $\delta \downarrow 0$, the expression in (4.16) has a finite explicit limit for $\beta$ a.e. with respect to Weiner measure. 
First, from (4.14) B), we note that as $\delta \rightarrow 0$, in the sense of tempered distributions,

$$
F_{\delta}^{\tilde{\varepsilon}}(x) \rightarrow F^{\tilde{\varepsilon}}(x)= \begin{cases}C(\tilde{\varepsilon})|x|^{\tilde{\varepsilon}-2}, & 0<\tilde{\varepsilon}<2, \tilde{\varepsilon} \neq 1 \\ C(1) \delta(x), & \tilde{\varepsilon}=1\end{cases}
$$

with $C(\tilde{\varepsilon})=-2(2 \pi)^{-1 / 2} \sin \left(\frac{(1-\tilde{\varepsilon}) \pi}{2}\right) \Gamma(2-\tilde{\varepsilon})$ and $C(1)=(2 \pi)^{-1 / 2}$. In (4.17) we utilize the fact that the inverse Fourier transform of $|k|^{1-\tilde{\varepsilon}}$ is $C(\tilde{\varepsilon})|x|^{\tilde{\varepsilon}-2}$ ([10]). From (4.17) we see that for every $\phi \in C_{0}^{\infty}\left(R^{1}\right)$

$$
\lim _{\delta \rightarrow 0} \int F_{\delta}^{\tilde{\varepsilon}}(x) \phi(x) d x=(F \tilde{\varepsilon}, \phi)
$$

with $\left(F^{\tilde{\varepsilon}}, \phi\right)$ given by

$$
\left(F^{\tilde{\varepsilon}}, \phi\right)= \begin{cases}C(\tilde{\varepsilon}) \int \phi(x)|x|^{\tilde{\varepsilon}-2} d x, & 1<\tilde{\varepsilon}<2 \\ C(\tilde{\varepsilon}) \int(\phi(x)-\phi(0))|x|^{\tilde{\varepsilon}-2} d x, & 0<\tilde{\varepsilon}<1\end{cases}
$$

In order to treat the limit in (4.16) we need to enlarge the limiting formula in (4.18) to a broader class of test functions, $\phi$. To do this, we recall that the Sobolev space $H^{s}\left(R^{1}\right)$ consists of functions $\varphi$ so that $|\hat{\varphi}|\left(1+|k|^{2}\right)^{s / 2} \in L^{2}\left(R^{1}\right)$ while $F e^{-1}\left(L^{\infty}\right)$ denotes the space of functions $\varphi$ so that $\hat{\varphi}$ is continuous and $\hat{\varphi} \in L^{\infty}$. We have the following

Lemma 4.1. Assume that the function $\varphi$ belongs to $H^{3 / 2-\gamma} \cap F e^{-1}\left(L^{\infty}\right)$ for all $\gamma>0$, then

$$
\lim _{\delta \rightarrow 0} \int \varphi F_{\delta}^{\tilde{\varepsilon}}(x) d x=\left(F^{\tilde{\varepsilon}}, \varphi\right) \text { for } \quad 0<\tilde{\varepsilon}<2
$$

Furthermore, there exists $\gamma(\tilde{\varepsilon})>0$ so that for $\delta \geqq 0$,

$$
\left|\left(F_{\delta}^{\tilde{\varepsilon}}, \varphi\right)\right| \leqq C\left(\|\varphi\|_{H^{3 / 2-\gamma}}+\|\hat{\varphi}\|_{L^{\infty}(|k| \leqq 1)}\right)
$$

with $C$ independent of $\delta$.

The proof of Lemma 4.1 is easy. First, (4.18) and the estimate in (4.20) B) combined with a standard approximation argument implies (4.20) A). Thus it is sufficient to establish (4.20) B) for $\varphi \in C_{0}^{\infty}\left(R^{1}\right)$. By Planchel's theorem, for $\delta \geqq 0$,

$$
\begin{aligned}
\left|\left(F_{\delta}^{\tilde{\varepsilon}}(x), \varphi\right)\right|= & \left|\int \hat{F}_{\delta}^{\varepsilon}(k) \hat{\varphi}(k) d k\right| \leqq\|\hat{\varphi}\|_{L^{\infty}} \int_{|k| \leqq 1}|k|^{1-\tilde{\varepsilon}} d k \\
& +\|\varphi\|_{H^{3 / 2-\gamma}}\left(\int_{|k| \geqq 1}|k|^{-1-2(\tilde{\varepsilon}-\gamma)}\right)^{1 / 2} .
\end{aligned}
$$

By setting $\gamma=\tilde{\varepsilon} / 2$ and using the fact that $\tilde{\varepsilon}$ satisfies $0<\tilde{\varepsilon}<2$, we see that both integrals on the right-hand side of the above estimate are finite and the estimate in (4.20) B) is established. We remark that every function $\varphi$ which belongs to $H^{3 / 2-\gamma}$ for all $\gamma>0$ also belongs to the Holder space $C^{\alpha}\left(R^{1}\right)$ for any $\alpha<1$. This fact is proved in Lemma 4.2 at the end of this section. In particular, if $\varphi \in H^{3 / 2-\gamma}$ for all $\gamma$, as expected from Lemma 4.1,

$$
\left(F^{\tilde{\varepsilon}}, \varphi\right)=C(\tilde{\varepsilon}) \int \frac{\varphi(x)-\varphi(0)}{|x|^{2-\tilde{\varepsilon}}} d x
$$

is an absolutely convergent integral for $0<\tilde{\varepsilon}<1$. 
In order to renormalize (4.12) with the scaling in (4.13), what remains is to determine the limit from (4.16) given by

$$
\lim \int F_{\delta}^{\tilde{\varepsilon}}(x) d \mu_{\beta} \quad \text { as } \quad \delta \downarrow 0
$$

with the measure $\mu_{\beta}$ defined in (4.15). The following lemma combined with Lemma 4.1 enables us to achieve this.

Lemma 4.2. For $\beta$ a.e. with respect to Wiener measure, the Borel measure $\mu_{\beta}$ defined in (4.15) is abolutely continuous with respect to Lebesgue measure, i.e. $d \mu_{\beta}=\varphi_{\beta}(x) d x$. Furthermore, the function $\varphi_{\beta}(x)$ belongs to $H^{3 / 2-\gamma} \cap F e^{-1}\left(L^{\infty}\right)$ for any $\gamma>0$ and we have the estimates
A) $\left|\hat{\varphi}_{\beta}(k)\right| \leqq(2 \pi)^{-1 / 2}$,
B) $E\left[\left\|\varphi_{\beta}\right\|_{3 / 2-\gamma}^{2}\right] \leqq C_{\gamma}$ for any $\gamma>0$.

In particular, for $\beta$ a.e. with respect to Wiener measure, $\varphi_{\beta}$ belongs to $C^{\alpha}\left(R^{1}\right)$ for any $\alpha<1$.

The estimate in (4.21) A) is obvious from the definition of $\mu_{\beta}$ in (4.15); the proof of $B$ ) is more difficult and we postpone this until the end of this section.

Lemma 4.2 combined with Lemma 4.1 and the dominated convergence theorem enables us to determine the limit of $Y_{\delta}(\beta, t)$. We summarize the result we have proved in the following

Proposition 4.1. With the scaling, $\varrho(\delta)=\delta^{\frac{1}{1+\tilde{\varepsilon} / 2}}$ for $0<\tilde{\varepsilon}<2$, the function $Y_{\delta}(\beta, t)$ has the following limit as $\delta \rightarrow 0, \beta$ a.e. with respect to Wiener measure:

A) For $1<\tilde{\varepsilon}<2$,

$$
\lim _{\delta \phi 0} Y_{\delta}(\beta, t)=\left(v_{0}\right)^{\tilde{\varepsilon} / 2-1} \frac{t^{1+\tilde{\varepsilon} / 2}}{2}(2 \pi)^{-1 / 2} C(\tilde{\varepsilon}) \int_{0}^{1} \int_{0}^{1}\left|\beta(s)-\beta\left(s^{\prime}\right)\right|^{2-\tilde{\varepsilon}} d s d s^{\prime} .
$$

B) For $0<\tilde{\varepsilon}<1$,

$$
\lim _{\delta \downarrow 0} Y_{\delta}(\beta, t)=\left(v_{0}\right)^{\tilde{z} / 2-1} \frac{t^{1+\tilde{\varepsilon} / 2}}{2}(2 \pi)^{-1 / 2} C(\tilde{\varepsilon}) \int \frac{\varphi_{\beta}(x)-\varphi_{\beta}(0)}{|x|^{2-\tilde{\varepsilon}}} d x .
$$

C) For $\tilde{\varepsilon}=1$

$$
\lim _{\delta \downarrow 0} Y_{\delta}(\beta, t)=\left(v_{0}\right)^{-1 / 2} \frac{t^{3 / 2}}{2}(2 \pi)^{-1} \varphi_{\beta}(0),
$$

where $\varphi_{\beta}(x)$ is the $\alpha$-Holder continuous function for any $\alpha<1$ determined from the definition

D)

$$
\int g(x) \varphi_{\beta}(x) d x=\int_{0}^{1} \int_{0}^{1} g\left(\beta(s)-\beta\left(s^{\prime}\right)\right) d s d s^{\prime}
$$

for all continuous functions $g$. 
Explicit Averaged Equations for $0<\tilde{\varepsilon}<2$ with Random Diffusivity. By utilizing Proposition 4.1 together with the general strategy sketched in (3.14)-(3.17), we obtain the following limiting representation formula as $\delta \downarrow 0$ for the ensemble averaged, $\langle T\rangle$, in (4.2) with the time scaling $\varrho(\delta)=\delta \frac{1}{\delta^{1+\varepsilon / 2}}$ :

$$
\begin{aligned}
\bar{T}(x, y, t) & =\lim _{\delta \downarrow 0}\left\langle T^{\delta}\left(\frac{x}{\delta}, \frac{y}{\delta}, \frac{t}{\varrho^{2}(\delta)}\right)\right\rangle \\
& =(2 \pi)^{-1} \iint e^{i(x \eta+y \xi)} \hat{T}_{0}(\xi, \eta) E\left[e^{-\xi^{2} t^{1+\varepsilon / 2 \mathscr{D} F^{\varepsilon}(\beta)}}\right] d \xi d \eta .
\end{aligned}
$$

The function $F^{\tilde{\varepsilon}}(\beta)$ is the random variable given by

$$
F^{\tilde{\varepsilon}}(\beta)=\left(F^{\tilde{\varepsilon}}, \varphi_{\beta}\right)
$$

with $F^{\tilde{\varepsilon}}$ the explicit distribution in (4.17) for $0<\tilde{\varepsilon}<2$ and $\varphi_{\beta}$ the test function depending on $\beta$ defined in (4.15) and D) of Proposition 4.1. Here $\mathscr{D}\left(v_{0}\right)$ is the coefficient

$$
\mathscr{D}\left(v_{0}, \tilde{\varepsilon}\right)=\left(v_{0}\right)^{\tilde{\varepsilon} / 2-1}(8 \pi)^{-1 / 2} .
$$

Our objective here is to interpret the solution formula in (4.22) as an effective equation involving appropriate ensemble averages over a random viscosity. To achieve this, we let $v_{\tilde{\varepsilon}}(\alpha)$, the random diffusivity, be the distribution function of the random variable, $F^{\tilde{\varepsilon}}(\beta)$, i.e.

$$
v_{\tilde{\varepsilon}}(\alpha)=E\left[F^{\tilde{\varepsilon}}(\beta) \leqq \alpha\right] .
$$

At the end of this section after the proof of Lemma 4.2, we verify

\section{Lemma 4.3.}

$$
v_{\varepsilon}(\alpha) \text { vanishes for } \alpha \leqq 0 .
$$

With (4.25), the formula in (4.22) becomes

$$
\bar{T}(x, y, t)=\int_{0^{+}}^{\infty} T(x, y, t, \alpha) d v_{\tilde{\varepsilon}}(\alpha)
$$

with

$$
T(x, y, t, \alpha)=(2 \pi)^{-1} \iint e^{i(x \eta+y \xi)} \widehat{T}_{0}(\xi, \eta) e^{-\xi^{2} t^{1+\varepsilon} / 2 \mathscr{D} \alpha} d \xi d \eta .
$$

For fixed $\alpha>0, T(x, y, t, \alpha)$ satisfies the diffusion equation

$$
\frac{\partial T}{\partial t}=\left(1+\frac{\tilde{\varepsilon}}{2}\right) t^{\tilde{\varepsilon} / 2} \mathscr{D} \alpha T_{y y},\left.\quad T\right|_{t=0}=T_{0}(x, y)
$$

so that

$$
T(x, y, t, \alpha)=\int K\left(y-y^{\prime}, t, \alpha\right) T_{0}\left(x, y^{\prime}\right) d y^{\prime}
$$

with kernel given by

$$
K(y, t, \alpha)=(4 \pi)^{-1 / 2} t^{-1 / 2-\tilde{\varepsilon} / 4}(\mathscr{D} \alpha)^{-1 / 2} \exp \left\{\frac{-|y|^{2}}{4 \mathscr{D} \alpha t^{1+\tilde{\varepsilon} / 2}}\right\} .
$$


With (4.26) and (4.29), the large-scale and long-time ensemble averaged solution is given by

$$
\bar{T}(x, y, t)=\int K\left(y-y^{\prime} \cdot t\right) T_{0}\left(x, y^{\prime}\right) d y^{\prime}
$$

with the explicit Green's function,

$$
K(y, t)=\int_{0^{+}}^{\infty} K(y, t, \alpha) d v_{\tilde{\varepsilon}}(\alpha)
$$

The formulas in (4.26), (4.28), and (4.30) give the effective equation as an ensemble average over solutions of the simple local diffusion equations in (4.28) with a distribution of viscosities determined by the distribution function, $v_{\tilde{\varepsilon}}(\alpha)$. Naively, one might have anticipated an effective local diffusion equation with the form in (4.28) for the regime $0<\tilde{\varepsilon}<2$ with a fixed value of $\alpha$, say $\bar{\alpha}(\tilde{\varepsilon})$, determined by $\tilde{\varepsilon}$. Both the scaling law, $\varrho(\delta)=\delta \frac{1}{1+\tilde{\varepsilon} / 2}$ and the local diffusion equation in (4.28) scale appropriately in $\tilde{\varepsilon}$ in the limiting regimes $\tilde{\varepsilon} \downarrow 0$ and $\tilde{\varepsilon} \uparrow 2$ to match the scaling behavior and dependence on bare diffusivity, $v_{0}$, in (4.4) and (4.9) for $\tilde{\varepsilon}<0$ and $\tilde{\varepsilon}>2$ respectively. This guess is completely wrong!! Instead, for a fixed value of $\tilde{\varepsilon}$, the averaged solution involves an ensemble of such solutions with a random diffusivity determined by the distribution function, $v_{\tilde{\varepsilon}}(\alpha)$, defined through (4.23) and (4.25). The issue of whether the solution of the local diffusion in (4.28) with an appropriate value $\bar{\alpha}(\tilde{\varepsilon})$ is a good approximation to the solution in (4.30) depends on the variance of the distribution function, $d v_{\tilde{\varepsilon}}(\alpha)$. As $\tilde{\varepsilon} \nearrow 2$, the formulas in A) of Proposition 4.1 yield the fact that $d v_{\tilde{\varepsilon}}(\alpha) \nearrow \delta_{v_{0}}$, a local Dirac mass so for $\tilde{\varepsilon}$ near 2 the appropriate local diffusion equation in (4.28) with $\alpha=v_{0}$ yields a good approximation to the ensemble averaged equation. However, for general $\tilde{\varepsilon}$ with $0<\tilde{\varepsilon}<2$, we conjecture that $d v_{\tilde{\varepsilon}}$ has a rather large variance, increasing as $\tilde{\varepsilon}$ decreases to zero so the local diffusion equation in (4.28) yields a poor approximation to the large-scale, longtime dynamics. At least for $1<\tilde{\varepsilon}<2$, the distribution function in (4.25) can be readily computed numerically through Chorin's algorithms for accurate evaluation of Wiener integrals $([6,12])$. The authors report on a detailed comparison of such approximations in [2].

Proof of Lemma 4.2. Since $d \mu_{\beta}$ is a probability measure, it follows that $\left\|\hat{\mu}_{\beta}\right\|_{L^{\infty}} \leqq 1$. We note that

$$
\hat{\mu}_{\beta}(k)=\frac{1}{\sqrt{2 \pi}} \int_{0}^{1} \int_{0}^{1} e^{i k\left(\beta(s)-\beta\left(s^{\prime}\right)\right)} d s d s^{\prime}=\frac{1}{\sqrt{2 \pi}}\left|\int_{0}^{1} e^{i k \beta(s)} d s\right|^{2} .
$$

Thus,

$$
\left\|\mu_{\beta}\right\|_{H^{3 / 2-\gamma}}^{2}=\frac{1}{2 \pi} \int\left(1+k^{2}\right)^{3 / 2-\gamma}\left|\int_{0}^{1} e^{i k \beta(s)} d s\right|^{4} d k,
$$

and, taking the expectation value,

$$
E\left(\left\|\mu_{\beta}\right\|_{H^{3 / 2-\gamma}}^{2}\right)=\frac{1}{2 \pi} \int\left(1+k^{2}\right)^{3 / 2-\gamma} E\left|\int_{0}^{1} e^{i k \beta(s)} d s\right|^{4} d k .
$$


We claim that $E\left|\int_{0}^{1} e^{i k \beta(s)} d s\right|^{4}$ decays like $k^{-4}$, for large $k$. To see this, we use Ito's formula [19] and write

$$
e^{i k \beta(1)}-1=i k \int_{0}^{1} e^{i k \beta(s)} d \beta(s)-\frac{k^{2}}{2} \int_{0}^{1} e^{i k \beta(s)} d s,
$$

whereby

$$
\left|\int_{0}^{1} e^{i k \beta(s)} d s\right| \leqq \frac{2}{k^{2}}\left|e^{i k \beta(s)}-1\right|+\frac{2}{k}\left|\int_{0}^{1} e^{i k \beta(s)} d \beta(s)\right| .
$$

Using the inequality $(a+b)^{4} \leqq 8\left(a^{4}+b^{4}\right)$, this implies that

$$
\left|\int_{0}^{1} e^{i k \beta(s)} d s\right|^{4} \leqq \frac{C_{1}}{k^{8}}+\frac{C_{2}}{k^{4}}\left|\int_{0}^{1} e^{i k \beta(s)} d \beta(s)\right|^{4},
$$

and thus

$$
E\left|\int_{0}^{1} e^{i k \beta(s)} d s\right|^{4} \leqq \frac{C_{1}}{k^{8}}+\frac{C_{2}}{k^{4}} E\left|\int_{0}^{1} e^{i k \beta(s)} d \beta(s)\right|^{4} \leqq \frac{C}{k^{4}}, \quad k>1,
$$

where $C$ is a numerical constant. This bound can be used to estimate the righthand side of (4.31). We have for all $\gamma>0$,

$$
E\left(\left\|\mu_{\beta}\right\|_{H^{3 / 2-}{ }_{\gamma}}^{2} \leqq 1+2 C \int_{1}^{\infty} \frac{\left(1+k^{2}\right)^{3 / 2-\gamma}}{k^{4}} d k<\infty,\right.
$$

and hence

$$
\left\|\mu_{\beta}\right\|_{H^{3 / 2-\gamma}}<\infty, \quad \beta \text { almost surely . }
$$

This implies that $\mu_{\beta}$ has a density in $C^{\alpha}$, for all $\alpha \in(0,1)$, by virtue of the embedding theorem $([1])$

$$
H^{3 / 2-\gamma}(\mathbf{R}) \subset C^{\alpha}(\mathbf{R}), \quad 0<\alpha<1-2 \gamma, \quad 0<\gamma<1 / 2 .
$$

Proof of Lemma 4.3. Since

$$
F^{\varepsilon}(\beta)=(2 \pi)^{-1 / 2} \int|k|^{1-\tilde{\varepsilon}}\left|\int_{0}^{1} e^{i k \cdot \beta(s)} d s\right|^{2} d k
$$

(where the integral converges for almost all $\beta$ ), $F^{\varepsilon}(\beta)$ is non-negative with probability one and, for all $\alpha<0, v_{\widetilde{\varepsilon}}(\alpha)=0$. We claim that $v_{\tilde{\varepsilon}}(0)=0$ as well. In fact, if $F^{\varepsilon}(\beta)=0$, then $\int_{0}^{1} e^{i k \cdot \beta(s)} d s=0$ for almost all $k \in \mathbf{R}$ and hence for all $k$. In particular

$$
\int_{0}^{1} \beta^{2}(s) d s=-\left.\frac{d^{2}}{d k^{2}} \int_{0}^{1} e^{i k \cdot \beta(s)} d s\right|_{k=0}=0 .
$$

Therefore,

$$
v_{\tilde{\varepsilon}}(0)=\operatorname{Pr}\left\{F^{\varepsilon}(\beta) \leqq 0\right\} \leqq\left\{\sup _{0 \leqq s \leqq 1}|\beta(s)|=0\right\}=0,
$$

and the claim is proved. 


\section{Renormalization with Time Dependent Velocity Fields}

Here we study renormalization for the model problem,

$$
\frac{\partial T}{\partial t}+v_{\delta}(x, t) \frac{\partial T}{\partial y}=\frac{1}{2} v_{0} \Delta T,\left.\quad T\right|_{t=0}=T_{0}(\delta x, \delta y),
$$

where $v_{\delta}(x, t)$ is the unsteady velocity field with stationary Gaussian statistics described in (2.15). This problem exhibits a remarkable range of different phenomena in renormalization when the positive parameter $z$ is varied. From the identity in (3.12), $a|k|^{z}$, measures the correlation time of the velocity statistitics at wave number $k$. In computing averaged equations for the large scale and long-time behavior of the solutions of (5.1), wave numbers with $|k| \ll 1$ are the significant range of values. If $z$ is a small positive number there is more rapid decorrelation in time at low wave numbers while stronger correlations in time develop at a fixed low wave number as $z$ increases. This intuitive fact underlies the trends for the results which we present in this section. There is an abrupt transition in the behavior at $z=2$ in the hyperscaling renormalized regimes where the time scaling function $\varrho(\delta)$ satisfies $\delta / \varrho(\delta) \downarrow 0$ as $\delta \downarrow 0$. For $z<2$, there is sufficiently rapid decorrelation in time at low wave numbers so that the renormalization theory for (4.1) coincides with the renormalization for the inviscid problem

$$
\frac{\partial T}{\partial t}+v_{\delta}(x, t) \frac{\partial T}{\partial y}=0,\left.\quad T\right|_{t=0}=T_{0}(\delta x, \delta y)
$$

in hyperscaling regimes. Nevertheless, the exact renormalization of (5.1) for $z<2$ exhibits some very rich behavior with three distinct anomalous scaling regimes for $z$ with $1<z<2$ and an abrupt transition to only two anomalous scaling regimes for $0<z \leqq 1$. For $z>2$, in hyperscaling regimes there is strong enough temporal correlation at low wave numbers so that the exact renormalization theory for (5.1) coincides with the renormalization theory for steady velocity fields, $v_{\delta}(x)$, which we discussed completely in Sect. 4. For the transition value, $z=2$, the scaling functions for the renormalization theory coincide with those for steady velocity fields from Sect. 4 but the formulas for the random diffusivity in the regime $0<\tilde{\varepsilon}<2$ are different and reflect the temporal statistics.

In the case of (5.1), the general representation fromula for the ensemble average over velocity statistics is given by

$$
\begin{aligned}
\bar{T}(x, y, t)= & \lim _{\delta \downarrow 0}\left\langle T^{\delta}\left(\frac{x}{\delta}, \frac{y}{\delta}, \frac{t}{\varrho^{2}(\delta)}\right)\right\rangle=(2 \pi)^{-1} \iint e^{i(x \eta+y \xi)} e^{-\frac{v_{0}}{2} \frac{\delta}{2}^{2} \xi^{2} t} \\
& \times \widehat{T}_{0}(\xi, \eta) E\left[e^{i \frac{\delta}{\varrho} \eta\left(v_{0}\right)^{1 / 2} \beta(1)} e^{-\xi^{2} Y_{\delta}(\beta, t)}\right] d \xi d \eta
\end{aligned}
$$

with

$$
Y_{\delta}(\beta, t)=\frac{\delta^{2}}{\varrho^{4}} \frac{t^{2}}{2} \int_{0}^{1} \int_{0}^{1} R_{\delta}\left(\frac{\left(v_{0} t\right)^{1 / 2}}{\varrho}\left(\beta(s)-\beta\left(s^{\prime}\right)\right), \frac{t}{\varrho^{2}}\left(s-s^{\prime}\right)\right) d s d s^{\prime}
$$

and

$$
R_{\delta}(\hat{x}, \widehat{t})=(2 \pi)^{-1} \int e^{i \hat{x} k} e^{-a|k| z|\hat{t}|} \psi_{0}\left(\frac{|k|}{\delta}\right) \psi_{\infty}(|k|)|k|^{1-\tilde{\varepsilon}} d k
$$


We remark that the representation formula for the inviscid case is obtained by setting $v_{0}=0$ in (5.3), (5.4), and (5.5).

The Regime of Mean Field Theory. Mean field behavior occurs when the standard long-time diffusive scaling $\varrho(\delta)=\delta$ and a local diffusion equation describe the large-scale and long-time behavior of the ensemble average. For the model problem in (5.1) the region of mean field theory is described by the two sets of inequalities, $\tilde{\varepsilon}<0$ for $z \geqq 2$ and $\tilde{\varepsilon}<2-z$ for $0<z<2$. To motivate the formulas in this regime, we recall that the particle displacement along the $y$-axis of a Brownian particle initially located at the origin with the velocity $v_{\delta}(x, t)$ from $(5.1)$ is given in the rescaled space and time by

$$
Z(\beta, \omega, t)=\delta v_{0}^{1 / 2} \beta\left(\frac{t}{\varrho^{2}}\right)+\delta \int_{0}^{t / \varrho^{2}} v_{\delta}(\beta(s), s) d s .
$$

We set $\varrho=\delta$ for standard diffusive scaling and calculate the mean-square particle displacement,

$$
E\left[\left\langle Z^{2}(\beta, \omega, t)\right\rangle\right]=\frac{v_{0}}{2} t+E\left[Y_{\delta}(\beta, t)\right]
$$

With $\varrho=\delta$, we compute that

$$
\lim _{\delta \rightarrow 0} E\left[Y_{\delta}(\beta, t)\right]=\frac{t}{4 \pi} \int\left(\frac{v_{0}}{2}|k|^{2}+a|k|^{z}\right)^{-1} \psi_{\infty}(|k|)|k|^{1-\tilde{\varepsilon}} d k .
$$

The integral in (5.8) is finite for $\tilde{\varepsilon}<0$ with $z \geqq 2$ and for $\tilde{\varepsilon}<2-z$ with $z$ satisfying $0<z<2$.

With the scaling for the particle displacement along the $y$-axis from (5.7) and (5.8), we anticipate that the effective averaged equation in the mean-field regime is given by

$$
\frac{\partial \bar{T}}{\partial t}=\frac{1}{2} v_{0} \Delta \bar{T}+D(\tilde{\varepsilon}) \bar{T}_{y y}
$$

with

$$
D(\tilde{\varepsilon})=\pi^{-1} \int\left(\frac{\nu_{0}}{2}|k|^{2}+a|k|^{z}\right)^{-1} \psi_{\infty}(|k|)|k|^{1-\tilde{\varepsilon}} d k
$$

An elementary proof of this fact is presented in the appendix of this paper.

5A) Regions with Renormalization where the Effects of Brownian Motion are Negligible. There are three distinct regions of anomalous hyperscaling, regions II, III, and IV depicted in Fig. 1, where Brownian motion is negligible. It is useful to begin with some general remarks which indicate that all of the computations presented here are based on systematic principles.

We recall that under the general frequency rescaling, $k^{\prime}=k / g(\delta), Y_{\delta}(\beta, t)$ from (5.4) is given by

$$
Y_{\delta}(\beta, t)=\frac{g(\delta)^{2-\tilde{\varepsilon}} \delta^{2}}{\varrho^{4}}(2 \pi)^{-1} \frac{t^{2}}{2} \int H_{\delta}(k, \beta) \psi_{0}\left(\frac{g(\delta)}{\delta}|k|\right) \psi_{\infty}(g(\delta) k)|k|^{1-\tilde{\varepsilon}} d k
$$


with

$$
H_{\delta}(k, \beta)=\int_{0}^{1} \int_{0}^{1} e^{i \frac{g(\delta)}{\varrho}\left(v_{0} t\right)^{1 / 2}\left(\beta(s)-\beta\left(s^{\prime}\right) k\right)} e^{-\frac{a t}{\varrho^{2}(g(\delta))^{z}|k| z\left|s-s^{\prime}\right|}} d s d s^{\prime} .
$$

Formally, in the regime where Brownian motion is negligible, the nonlinear scaling functions $g(\delta), \varrho(\delta)$ satisfy

$$
\frac{g(\delta)}{\varrho(\delta)} \downarrow 0 \quad \text { as } \quad \delta \downarrow 0 .
$$

The function $H_{\delta}(k, \beta)$ from (5.11) satisfies the bound

$$
\left|H_{\delta}(k, \beta)\right| \leqq \bar{H}_{\delta}(k) \equiv \int_{0}^{1} \int_{0}^{1} e^{-\frac{a t}{\varrho^{2}(g(\delta))^{z}|k| z\left|s-s^{\prime}\right|}} d s d s^{\prime}=G\left(a t|k|^{z} \frac{(g(\delta))^{z}}{\varrho^{2}}\right)
$$

with $G(s)=s^{-1}-s^{-2}\left(1-e^{-s}\right)$. The function $G(s)$ has the elementary properties
A) $G(s) \rightarrow 1$ as $s \downarrow 0$,
B) $|G(s)| \leqq C(1+|s|)^{-1}$,
C) $\left|G(s)-s^{-1}\right| \leqq C|s|^{-2}$ for $|s| \geqq 1$.

We define $\bar{Y}_{\delta}(t)$ as given by

$$
\bar{Y}_{\delta}(t)=\frac{g(\delta)^{2-\tilde{\varepsilon}} \delta^{2}}{\varrho^{4}}(2 \pi)^{-1} \frac{t^{2}}{2} \int\left[\delta(k) \psi_{0}\left(\frac{g(\delta)}{\delta}|k|\right) \psi_{\infty}(g(\delta) k)|k|^{1-\tilde{\varepsilon}} d k\right.
$$

The representation formula for the inviscid problem in (5.2) is given by

$$
(2 \pi)^{-1} \iint e^{i(x \eta+y \xi)} e^{-\xi^{2} \bar{Y}_{\delta}(t)} \widehat{T}_{0}(\eta, \xi) d \eta d \xi .
$$

Summarizing (5.10)-(5.16), as well as (3.14)-(3.19), we see that Brownian motion is negligible and the renormalization theory for (5.1) coincides with the renormalization theory for the inviscid problem in (5.2) provided that
A) $\delta / \varrho(\delta) \downarrow 0$ as $\delta \downarrow 0$,
B) $\bar{Y}_{\delta}(t) \rightarrow \bar{Y}(t)$ as $\delta \downarrow 0$,
C) $\bar{Y}_{\delta}(t)-Y_{\delta}(\beta, t) \rightarrow 0$ as $\delta \downarrow 0$ for fixed $\beta$.

By Taylor's theorem applied to $e^{i s}$, we see that

$$
\left|H_{\delta}(k, \beta)-\bar{H}_{\delta}(k)\right| \leqq 2 \frac{g(\delta)}{\varrho}\left(v_{0} t\right)^{1 / 2}|k| \max _{0<s<1} \mid \beta(s) \bar{H}_{\delta}(k) .
$$

From (5.17) and (5.18), in any regime where $g(\delta) / \varrho \rightarrow 0$, we have

$$
\left|H_{\delta}(k, \beta)-\bar{H}_{\delta}(k)\right| \leqq\left(\frac{g(\delta)}{\varrho}\right)^{1 / 2} C_{\beta} \bar{H}_{\delta}(k) \text { for }|k| \leqq(\varrho / g(\delta))^{1 / 2} .
$$

Below we will always pick the nonlinear rescaling functions, $g(\delta), \varrho(\delta)$ so that $\bar{Y}_{\delta}(t)$ given in (5.15) satisfies the hypothesis of the dominated convergence theorem with limit $\bar{Y}(t)$ as $g(\delta) / \varrho \downarrow 0$; from (5.13), (5.18), and (5.19), we see that $Y_{\delta}(\beta, t)$ will always satisfy the same hypotheses for the dominated convergence theorem and 
furthermore,

$$
\lim _{\delta \rightarrow 0} Y_{\delta}(\beta, t)=\lim \bar{Y}_{\delta}(t)=\bar{Y}(t)
$$

The distinct anomalous scaling regions, II, III, and IV depicted in Fig. 1 are distinguished by the behavior of $g(\delta)^{z} / \varrho^{2}$. In particular,
A) $g(\delta)^{z} / \varrho^{2} \nearrow \infty$ as $\delta \downarrow 0$ in region II,
B) $g(\delta)^{z} / \varrho^{2} \downarrow 0$ as $\delta \downarrow 0$ in region III,
C) $g(\delta)^{z} / \varrho^{2} \equiv 1$ in region IV.

Renormalization in Region II. Region II is defined by the inequalities, $2-z<\tilde{\varepsilon}<4-2 z$ with $z<2$. In this region we set $g(\delta)=\delta$ in (5.15), then

$$
\bar{Y}_{\delta}(t)=\frac{\delta^{4-\tilde{z}-z} t}{\varrho^{2}} \frac{t}{a}(4 \pi)^{-1} \int \psi_{0}(|k|) \psi_{\infty}(\delta|k|)|k|^{1-\varepsilon-z}\left[1-\frac{\left(1-e^{-a t \frac{\delta^{z}}{\varrho^{2}}|k|^{z}}\right)}{a t \frac{\delta^{z}}{\varrho^{2}}|k|^{z}}\right] d k .
$$

The formula in (5.22) suggests the scaling function,

$$
\varrho(\delta)=\delta^{\frac{4-\tilde{\varepsilon}-z}{2}} .
$$

We compute that

$$
\delta^{z} / \varrho^{2} \nearrow \infty \text { as } \delta \downarrow 0 \text { if and only if } \tilde{\varepsilon}<4-2 z
$$

while

$$
\int \psi_{0}(|k|)|k|^{1-\tilde{\varepsilon}-z} d k<\infty \quad \text { if and only if } \tilde{\varepsilon}>2-z .
$$

With (5.22)-(5.24), the general principle presented above (5.20), and the dominated convergence theorem, in region II with the anomalous scaling $\varrho(\delta)=\delta^{\frac{4-\varepsilon-z}{2}}$, the ensemble averaged effective diffusion equation for (5.1) is given by

$$
\frac{\partial \bar{T}}{\partial t}=D(\tilde{\varepsilon}, z) \bar{T}_{y y},\left.\quad \bar{T}\right|_{t=0}=T_{0}(x, y)
$$

with

$$
D(\tilde{\varepsilon}, z)=(4 \pi a)^{-1} \int \psi_{0}(|k|)|k|^{1-\tilde{\varepsilon}-z} d k
$$

for $2-z<\tilde{\varepsilon}<4-2 z$ and $z<2$.

Renormalization in Region III. Region III is defined by the inequalities, $4-2 z<\tilde{\varepsilon}<4$ for $z<2$ and $2<\tilde{\varepsilon}<4$ for $z \geqq 2$. In this region the scaling function for time and the effective diffusion equation are given by (4.7) and (4.9) from the steady case already discussed in Sect. 4 . With $g(\delta)=\delta$ in (5.15), we have

$$
\bar{Y}_{\delta}(t)=\frac{\delta^{4-\varepsilon}}{\varrho^{4}}(2 \pi)^{-1} \frac{t^{2}}{2} \int G\left(a t|k|^{z} \frac{\delta^{z}}{\varrho^{2}}\right) \psi_{0}(|k|) \psi_{\infty}(\delta|k|)|k|^{1-\tilde{\varepsilon}} d k
$$


With the scaling $\varrho=\delta^{1-\tilde{z} / 4}$,

$$
\delta^{z} / \varrho^{2} \rightarrow 0 \text { if and only if } \tilde{\varepsilon}>4-2 z,
$$

so that

$$
G\left(a t|k|^{2} \frac{\delta^{z}}{\varrho^{2}}\right) \rightarrow 1 \quad \text { as } \quad \delta \downarrow 0
$$

while

$$
\int \psi_{0}(k)|k|^{1-\tilde{\varepsilon}} d k<\infty \text { for } \tilde{\varepsilon}>2 \text {. }
$$

With (5.22)-(5.24), the general principle presented above (5.20), and the dominated convergence theorem, in region III the effective diffusion equation is given by

$$
\frac{\partial \bar{T}}{\partial t}=t D(\tilde{\varepsilon}) \bar{T}_{y y},\left.\quad \bar{T}\right|_{t=0}=T_{0}(x, y)
$$

with

$$
D(\tilde{\varepsilon})=(2 \pi)^{-1} \int \psi_{0}(|k|)|k|^{1-\varepsilon} d k .
$$

Remark. An interesting facet of the renormalization theory for regions II and III is the fact that the scaling functions in (5.23) and (4.7) for regions II and III both agree at the common boundary $\tilde{\varepsilon}=4-2 z$ for $z<1$ with $\varrho(\delta)=\delta^{z / 2}$ but the coefficients for effective diffusivity are discontinuous across this boundary. To display the source of this discontinuity, we give the large-scale, long-time averaged diffusion equation at these boundary values. With time scaling function, $\varrho(\delta)=\delta^{z / 2}$ we have the effective diffusion equation

$$
\frac{\partial \bar{T}}{\partial t}=D(t, z) \bar{T}_{y y}
$$

for $\tilde{\varepsilon}=4-2 z$ and $z<1$ with

$$
D(t, z)=(4 \pi a)^{-1} \int \psi_{0}(|k|)|k|^{1-\varepsilon-z}\left[1-\frac{\left(1-e^{-a t|k|^{z}}\right)}{a t|k|^{z}}\right] d k .
$$

We see that $D(t, z)$ satisfies $D(t, z)=t D(\tilde{\varepsilon})+0\left(t^{2}\right)$ with $D(\tilde{\varepsilon})$ given in (5.28) for small renormalized times while $D(t, z)=D(\tilde{\varepsilon}, z)+0\left(\frac{1}{t}\right)$ as $t \nearrow \infty$ with $D(\tilde{\varepsilon}, z)$ given in (5.25); thus at this critical value with $\tilde{\varepsilon}=4-2 z$, solutions of the effective equation behave like those in Region III at short renormalized times and like those in Region II at large renormalized times with the time rescaling $\varrho=\delta^{z / 2}$.

Renormalization in Region IV. Region IV is defined by the inequalities $4-2 z$ $<\tilde{\varepsilon}<2$ for $1<z<2$. In this region, we utilize the scaling function $g(\delta)$ with $g(\delta)$ $=\varrho^{2 / z}(a t)^{-1 / z}$ so that $a t(g(\delta))^{z} \varrho(\delta)^{-1}=1$. Since $g(\delta) / \varrho(\delta)=\varrho^{2 / z-1}(a t)^{-1 / 2}$, we see that $g(\delta) / \varrho(\delta) \downarrow 0$ as $\delta \downarrow 0$ provided that $z<2$ so that the effects of Brownian motion are negligible for $z<2$. We use this choice for $g(\delta)$ in (5.15) and obtain

$$
\bar{Y}_{\delta}(t)=\varrho^{\frac{4-2 \varepsilon}{z}-4} \delta^{2}(4 \pi)^{-1} a^{\frac{z-2}{z}} t^{2+\frac{\varepsilon-2}{z}} \int G\left(|k|^{z}\right) \psi_{0}\left(\frac{g(\delta)}{\delta}|k|\right) \psi_{\infty}(g(\delta) k)|k|^{1-\tilde{\varepsilon}} d k
$$


We choose $\varrho(\delta)$ so that the first term on the right-hand side of $(5.31)$ has value one, i.e.

$$
\varrho(\delta)=\delta^{z / 2}\left(\frac{1}{\delta^{z-1+\varepsilon / 2}}\right)
$$

We compute that

$$
\frac{g(\delta)}{\delta} \nearrow \infty \quad \text { as } \quad \delta \downarrow 0 \quad \text { if and only if } \quad \tilde{\varepsilon}>4-2 z
$$

so that the infrared cut-off is negligible provided that $\tilde{\varepsilon}>4-2 z$. From (5.22)-(5.24), the general principle presented above (5.20) and the dominated convergence theorem, the averaged diffusion equation with the hyperscaling in (5.32) is given by

$$
\frac{\partial \bar{T}}{\partial t}=t^{1+\frac{\tilde{\varepsilon}-2}{z}} D(\tilde{\varepsilon}, z) \bar{T}_{y y}
$$

with

$$
D(\tilde{\varepsilon}, z)=(4 \pi)^{-1}\left(2+\frac{\tilde{\varepsilon}-2}{z}\right) a \frac{\tilde{\varepsilon}-2}{2} \int|k|^{1-\tilde{\varepsilon}}|k|^{-z}\left[1-\frac{\left(1-e^{-|k|^{z}}\right)}{|k|^{z}}\right] d k .
$$

Of course, this derivation relies on the fact that the integral on the right-hand side of (5.35) is finite. The required integral converges provided that

$$
\tilde{\varepsilon}<2 \text { for the local singularity near zero }
$$

and

$$
\tilde{\varepsilon}>2-z \text { for the behavior as }|k| \rightarrow \infty \text {. }
$$

The requirements in (5.33) and (5.36) lead to the restrictions $4-2 z<\tilde{\varepsilon}<2$ used in defining region IV besides $z<2$.

5B) The Regime $0<\tilde{\varepsilon}<2$ and $z \geqq 2-$ The Region with Random Nonlocal Diffusivity. In this regime, we have similar behavior as we described in Sect. 4C) for steady velocity fields. In fact, where the effects of Brownian motion are not negligible for $z>2$ and $0<\tilde{\varepsilon}<2$, the scaling law and ensemble averaged Green's function coincide exactly with those for the steady case in (4.13) and (4.30) respectively. For $z=2$, the same scaling law from (4.13) applies in the renormalization but the formulas for the random diffusivity are different. We will be terse in our discussion since most of the arguments parallel those in Sect. 4C).

The Case with $z=2$ and $0<\tilde{\varepsilon}<2$. With the same time scaling law from (4.13), $\varrho(\delta)=\delta \frac{1}{1+\tilde{\varepsilon} / 2}$, we calculate as in (4.12) that $Y_{\delta}(\beta, t)$ from (5.4) is given by,

$$
Y_{\delta}(\beta, t)=\left(v_{0}\right)^{\varepsilon / 2-1} \frac{t^{1+\tilde{\varepsilon} / 2}}{2}(2 \pi)^{-1 / 2} \int \overline{\hat{\tilde{\varphi}}_{\beta}(k)} \widehat{F}_{\delta}^{\tilde{\varepsilon}}(k) d k
$$

with $\widehat{F}_{\delta}^{\varepsilon}(k)$ already defined in (4.14) and

$$
\widehat{\tilde{\varphi}}_{\beta}(k, t)=(2 \pi)^{-1 / 2} \int_{0}^{1} \int_{0}^{1} e^{i\left(\beta(s)-\beta\left(s^{\prime}\right)\right) k} e^{-\frac{a}{v_{0} t} k^{2}\left|s-s^{\prime}\right|} d s d s^{\prime} .
$$


Let $\tilde{\varphi}_{\beta}(x, t)$ be the function with Fourier transform given by $\hat{\tilde{\varphi}}_{\beta}(k, t)$. The function $\hat{\tilde{\varphi}}_{\beta}(k, t)$ is obviously continuous in $k$. We claim that for fixed $t, \tilde{\varphi}_{\beta}(x, t)$ belongs to $F e^{-1}\left(L^{\infty}\right) \cap H^{3 / 2-\gamma}(R)$ for any $\gamma>0$. We have the estimate,

$$
\left|\hat{\hat{\varphi}}_{\beta}(k, t)\right| \leqq(2 \pi)^{-1 / 2} G\left(\left(\frac{a}{v_{0} t}\right) k^{2}\right) \leqq(2 \pi)^{-1 / 2}\left(1+\frac{a}{v_{0} t} k^{2}\right)^{-1}
$$

with $G(s)$, the explicit function in (5.14). The estimates in (5.39) guarantee that $\tilde{\varphi}_{\beta}(k, t) \varepsilon \mathrm{Fe}^{-1}\left(L^{\infty}\right) \cap H^{3 / 2-\gamma}(R)$; Lemma 4.1 and Plancherel's theorem guarantee that

$$
\lim _{\delta \downarrow 0} \int \overline{\hat{\tilde{\varphi}}_{\beta}(k)} \hat{F}_{\delta}^{\varepsilon}(k) d k=\left(F^{\tilde{\varepsilon}}, \tilde{\varphi}_{\beta}\right)
$$

with $F^{\tilde{\varepsilon}}$ the distribution already defined in (4.17), (4.19). From (5.37) and (5.40), we obtain that

$$
\lim _{\delta \downarrow 0} Y_{\delta}(\beta, t)=\left(v_{0}\right)^{\tilde{\varepsilon} / 2-1} \frac{t^{1+\varepsilon / 2}}{2}(2 \pi)^{-1 / 2}\left(F^{\tilde{\varepsilon}}, \tilde{\varphi}_{\beta}\right) .
$$

It should be clear to the reader that we can repeat a similar discussion as given earlier in (4.23) - (4.30) to obtain a rigorous formula for the Green's function for the effective equation as an average of Green's functions for the simple diffusion problem in (4.28) with respect to the distribution function of the random variable, $\left(F^{\tilde{\varepsilon}}, \tilde{\varphi}_{\beta}\right)$. We omit the details. However, we do mention here that there is a simple explicit formula for $\tilde{\varphi}_{\beta}$ with Fourier transform from (5.38). In fact

$$
\begin{aligned}
\tilde{\varphi}_{\beta}(x)= & (4 \pi)^{-1 / 2}\left(\frac{a}{v_{0} t}\right)^{1 / 2} \int_{0}^{1} \int_{0}^{1}\left|s-s^{\prime}\right|^{-1 / 2} \\
& \times \exp \left(-\frac{v_{0} t}{4 a}\left|s-s^{\prime}\right| \mid x+\left(\beta(s)-\left.\beta\left(s^{\prime}\right)\right|^{2}\right) d s d s^{\prime} .\right.
\end{aligned}
$$

We record the formula in (5.42) because it is potentially useful for the numerical evaluation of the distribution function for $\left(F^{\tilde{\varepsilon}}, \tilde{\varphi}_{\beta}\right)$ (see $\left.[6,12]\right)$. We discuss the analogue of Lemma 4.3 for this problem at the end of this section.

The Case with $z>2$ and $0<\tilde{\varepsilon}<2$. Once again with the scaling law from (4.13), $\varrho(\delta)$ $=\delta \frac{1}{1+\tilde{\varepsilon} / 2}$, we calculate that $Y_{\delta}(\beta, t)$ from (5.4) is given by

$$
Y_{\delta}(\beta, t)=\left(v_{0}\right)^{\tilde{z} / 2-1} \frac{t^{1+\xi / 2}}{2}(2 \pi)^{-1 / 2} \sqrt{\hat{\varphi}_{\beta, \delta}} \hat{F}_{\delta}^{\varepsilon}(k) d k
$$

with $\widehat{F}_{\delta}^{\xi}(k)$ from (4.14) and

$$
\hat{\varphi}_{\beta, \delta}(k)=(2 \pi)^{-1 / 2} \int_{0}^{1} \int_{0}^{1} e^{i\left(\beta(s)-\beta\left(s^{\prime}\right)\right) k} e^{-A Q^{z-2}|k| z\left|s-s^{\prime}\right|} d s d s^{\prime}
$$

with $A=a t\left(v_{0} t\right)^{-z / 2}$. Since $z$ satisfies $z>2$, we have

$$
e^{-A e^{z-2|k| z\left|s-s^{\prime}\right|} \rightarrow 1} \quad \text { as } \quad \delta \downarrow 0
$$

and therefore,

$$
\lim _{\delta \rightarrow 0} \hat{\varphi}_{\beta, \delta}(k)=\hat{\varphi}_{\beta}(k)=(2 \pi)^{-1 / 2} \int_{0}^{1} \int_{0}^{1} e^{i\left(\beta(s)-\beta\left(s^{\prime}\right)\right) k} d s d s^{\prime}
$$


pointwise in $k$. Thus, we anticipate from (5.46) that

$$
\lim _{\delta \downarrow 0} \int \bar{\varphi}_{\beta, \delta} \hat{F}_{\delta}^{\varepsilon}(k) d k=\left(F^{\tilde{\varepsilon}}, \varphi_{\beta}\right) \text {. }
$$

If (5.47) is valid, then it follows by taking the limit as $\delta \downarrow 0$ in (5.43) that Proposition 4.1 is also valid for $z>2$. Thus, for $z>2$ and $\tilde{\varepsilon}$ with $0<\tilde{\varepsilon}<2$, the discussion in (4.23)-(4.30) remains valid and the large-scale, long-time ensemble averaged solution coincides with that derived for the steady case in Sect. 4 .

It remains to prove the identity in (5.47). The following lemma contains the technical ingredients needed to justify the formula in (5.47). The idea of the proof is similar to the one used in Lemma 4.3. However, the proof is more technical due to the need to obtain estimates uniform in $\delta$ on the $H^{3 / 2-\gamma}$ norm of $\varphi_{\beta, \delta}(x, t)$ defined in (5.44). This is needed in order to pass to the limit as $\delta \rightarrow 0$ for each path $\beta(\cdot)$ on a set of full Weiner measure.

Lemma 5.1. For each $z \geqq 2$, consider the family of auxiliary functions $\tilde{\varphi}_{\beta, \delta}(x, t)$, defined through their Fourier transform in $x, b y$

$$
\hat{\bar{\varphi}}_{\beta, \delta}(k, t)=\frac{1}{\sqrt{2 \pi}} \int_{0}^{1} \int_{0}^{1} e^{i k\left(\beta(s)-\beta\left(s^{\prime}\right)\right)-A(z, k, t)\left|s-s^{\prime}\right|} d s d s^{\prime},
$$

where

$$
A(z, k, t)=v_{0}^{-z / 2}\left(\frac{a t}{\varrho}\right)^{1-z / 2}|k|^{z}
$$

and

$$
\varrho(\delta)=\delta^{\frac{1}{1+\varepsilon / 2}}
$$

Then

(i) For each $\gamma \in\left(0, \frac{1}{2}\right]$, there exists a constant $C(\gamma)$ such that

$$
E\left\{\sup _{0 \leqq \delta \leqq 1}\left\|\varphi_{\beta, \delta}(\cdot, t)\right\|_{H^{3 / 2}-\gamma}^{2}\right\} \leqq C(\gamma)<\infty .
$$

In particular, for almost all $\beta$,

$$
\sup _{0 \leqq \delta \leqq 1}\left\|\varphi_{\beta, \delta}(\cdot, t)\right\|_{H^{3 / 2-\gamma}}<\infty
$$

(ii) If $z>2$,

$$
\lim _{\delta \downarrow 0} \tilde{\varphi}_{\beta, \delta}(x, t)=\varphi_{\beta}(x),
$$

where $\varphi_{\beta}$ is the density of the measure $d \mu_{\beta}$ of Sect. 4. Moreover

$$
\lim _{\delta \rightarrow 0}\left(F_{\delta}^{\mathfrak{z}}, \varphi_{\beta, \delta}(\cdot, t)\right)=\left(F^{\mathfrak{\varepsilon}}, \varphi_{\beta}\right)=F^{\mathfrak{\varepsilon}}(\beta) \text {. }
$$

(iii) For $z=2$,

$$
\operatorname{Prob}\left\{\beta:\left(F^{\tilde{\varepsilon}}, \tilde{\varphi}_{\beta}\right) \leqq 0\right\}=0
$$


Proof. (i) Clearly, $\left|\hat{\varphi}_{\beta, \delta}(k, t)\right| \leqq \frac{1}{\sqrt{2 \pi}}$ for all $k$. For $|k| \geqq 1$, set

$$
D(z, k, t)=\left(2 A(z, k, t) k^{-2}\right)^{1 / 2},
$$

so that

$$
\hat{\varphi}_{\beta, \delta}(k, t)=(2 \pi)^{-1 / 2} \int_{0}^{1} \int_{0}^{1} e^{i k\left(\beta(s)-\beta\left(s^{\prime}\right)\right)-1 / 2 D^{2}\left|s-s^{\prime}\right| k^{2}} d s d s^{\prime} .
$$

We introduce an auxiliary Brownian motion $\beta^{*}(s), O \leqq s \leqq 1$, independent of $\beta(s)$, and write

$$
\hat{\varphi}_{\beta, \delta}(k, t)=(2 \pi)^{\frac{-1}{2}} E^{\beta^{*}}\left|\int_{0}^{1} e^{i k\left(\beta(s)-D \beta^{*}(s)\right)} d s\right|^{2} .
$$

By Jensen's inequality,

$$
\begin{aligned}
\left|\hat{\varphi}_{\beta, \delta}(k, t)\right|^{2} & \leqq(2 \pi)^{-1} E^{\beta^{*}}\left|\int_{0}^{1} e^{i k \cdot\left(\beta(s)-D \beta^{*}(s)\right)} d s\right|^{4} \\
& =(2 \pi)^{-1} E^{\beta^{*}}\left|\int_{0}^{1} e^{i k \tilde{\beta}(s) \sqrt{1+D^{2}}} d s\right|^{4},
\end{aligned}
$$

where $\widetilde{\beta}(s)=\frac{\beta(s)-D \beta^{*}(s)}{\sqrt{1+D^{2}}}$, which is again a Brownian motion. The arguments of Lemma 4.2 imply for any Brownian motion $\widetilde{\beta}(s)$ the estimate

$$
\left|\int_{0}^{1} e^{i k \tilde{\beta}(s) \sqrt{1+D^{2}}} d s\right|^{4} \leqq \frac{C_{1}}{k^{8}\left(1+D^{2}\right)^{4}}+\frac{C_{2}}{k^{4}\left(1+D^{2}\right)^{2}}\left|\int_{0}^{1} e^{i k \tilde{\beta}(s) \sqrt{1+D^{2}}} d s\right|^{4},
$$

where $C_{1}$ and $C_{2}$ are numerical constants and $D=D(k, \delta, t)$ is given explicitly. Thus, we have

$$
\begin{aligned}
& E^{\beta}\left(\sup _{\delta \geqq 1}\left\|\varphi_{\beta}(\cdot, t, \delta)\right\|_{H^{3 / 2-\gamma}}^{2}\right) \\
& \left.\leqq\left. E^{\beta}\left\{\sup _{\delta \leqq 1} \int\left(1+k^{2}\right)^{3 / 2-\gamma} \mid \hat{\varphi}_{\beta}(k, t, \delta)\right)\right|^{2} d k\right\} \\
& \leqq E^{\beta} \int\left(1+k^{2}\right)^{3 / 2-\gamma}\left(\sup _{\delta \leqq 1}\left|\hat{\varphi}_{\beta}(k, t, \delta)\right|^{2}\right) d k \\
& \leqq E^{\beta} \int\left(1+k^{2}\right)^{3 / 2-\gamma} \sup _{\delta \leqq 1} E^{\beta^{*}}\left|\int_{0}^{1} e^{i k \tilde{\beta}(s) \sqrt{1+D^{2}}} d s\right|^{4} d k \\
& \leqq \int\left(1+k^{2}\right)^{3 / 2-\gamma} E^{\tilde{\beta}}\left[\sup _{\delta \leqq 1}\left|\int_{0}^{1} e^{i k \tilde{\beta}(s) \sqrt{1+D^{2}}} d s\right|^{2}\right] d k \\
& \leqq C_{3}+\int_{|k| \geqq 1}\left(1+k^{2}\right)^{3 / 2-\gamma} E^{\tilde{\beta}}\left[\left.\sup _{\delta \leqq 1}^{1} \int_{0}^{1 i k \tilde{\beta}(s) \sqrt{1+D^{2}}} d s\right|^{4}\right] d k \\
& \leqq C_{3}+C_{4} \int_{|k| \geqq 1} \frac{\left(1+k^{2}\right)^{3 / 2-\gamma}}{k^{4}} E^{\tilde{\beta}}\left[\sup _{\delta \leqq 1}\left|\int_{0}^{1} e^{i k \tilde{\beta}(s) \sqrt{1+D^{2}}} d \beta(s)\right|^{4}\right] d k \text {, }
\end{aligned}
$$


where (5.48) was used and $C_{3}$ and $C_{4}$ are numerical constants. We focus on the expectation value

$$
E \equiv E^{\tilde{\beta}}\left[\sup _{\delta \leqq 1}\left|\int_{0}^{1} e^{i \tilde{\beta} \tilde{\beta}(s) \sqrt{1+D^{2}}} d \beta(s)\right|^{4}\right]
$$

and argue that it is bounded by a constant depending on $\gamma$ but not $\delta$ or $k$. The estimate (5.48) and its consequence (5.49) follow immediately from this fact. To bound $E$, we make a new change of variables:

$$
\begin{aligned}
E & =E\left[\sup _{\delta \leqq 1}\left|\frac{1}{|k| \sqrt{1+D^{2}}} \int_{0}^{k^{2}\left(1+D^{2}\right)} e^{i \beta(s)} d \beta(s)\right|^{4}\right] \\
& =E\left[\sup _{\delta \leqq 1}\left|\frac{1}{\sqrt{T(\delta)}} \int_{0}^{T(\delta)} \sigma(s) d \beta(s)\right|^{4}\right],
\end{aligned}
$$

where $T(\delta)=k^{2}\left(1+D^{2}\right)$ and $\sigma(s)=e^{i \beta(s)}$. For simplicity, we assume in the following argument that $\sigma(s)$ denotes either the real or the imaginary part of $e^{i \beta(s)}$. Consider the natural time-scale

$$
M(t)=\int_{0}^{t} \sigma^{2}(s) d s
$$

of the martingale $\int_{0}^{t} \sigma(s) d \beta(s)$. It is well-known ([19]) that there exists a Wiener process $W(\cdot)$ such that

$$
\int_{0}^{t} \sigma(s) d \beta(s)=W[M(t)], \quad t>0 .
$$

Hence,

$$
\begin{aligned}
E & =E\left\{\sup _{\delta \leqq 1} \mid \frac{1}{T(\delta)^{1 / 2}} W\left[\left.M((T(\delta))]\right|^{4}\right\}\right. \\
& =E\left\{\sup _{\delta \leqq 1}\left(\frac{M(T(\delta))}{T(\delta)}\right)^{2}\left(\frac{W(M(T(\delta)))}{M(T(\delta))^{1 / 2}}\right)^{4}\right\} \\
& \leqq E\left\{\sup _{\delta \leqq 1}\left|\frac{W[M(T(\delta))]}{M(T(\delta))^{1 / 2}}\right|^{4}\right\} \\
& =E\left\{\sup _{k \leqq T_{0}}\left|\frac{W(t)}{t^{1 / 2}}\right|^{4}\right\},
\end{aligned}
$$

where

$$
T_{0} \equiv \sup _{\delta \leqq 1} T(\delta)=k^{2}+2 a v_{0}^{-\frac{z}{2}} t^{1-\frac{z}{2}}|k|^{z}
$$

and where we used that $M(t) \leqq t$, since $\|\sigma\|_{L^{\infty}} \leqq 1$. But, by the scaling property of Brownian motion,

$$
E\left\{\sup _{t \leqq T_{0}}\left|\frac{W(t)}{t^{1 / 2}}\right|^{4}\right\}=E\left\{\sup _{t \leqq 1}\left|\frac{W(t)}{t^{1 / 2}}\right|^{4}\right\}
$$


a constant independent of $k$ or $\delta$. This concludes the proof of item (i).

(ii) For $z>2, A(z, k, t)=a v_{0}^{-z / 2}\left(\frac{t}{\varrho(\delta)}\right)^{1-\frac{z}{2}}|k|^{z}$ converges to zero as $\delta \rightarrow 0$. Thus,

$$
\lim _{\delta \downarrow 0} \hat{\varphi}_{\beta, \delta}(k, t)=\hat{\varphi}_{\beta}(k),
$$

the Fourier transform of $\varphi_{\beta}$, defined in Sect. 4 with the convergence uniform on any interval, $|k| \leqq R$ with $R>0$. From (i), we know that on a set of full Wiener measure, the family $\left\{\tilde{\varphi}_{\beta, \delta}(x, t)\right\}_{\delta \leqq 1}$ is bounded in $H^{3 / 2-\gamma} \cap F e^{-1}\left(L^{\infty}\right)$ for all $\gamma>0$. Therefore, $\varphi_{\beta, \delta}$ converges to $\varphi_{\beta}$ in $H^{3 / 2-\gamma^{\prime}}$ for $\gamma^{\prime}>\gamma$ and any $\gamma>0$, i.e. $\left\|\varphi_{\beta, \delta}-\varphi_{\beta}\right\|_{H^{3 / 2}-\gamma^{\prime}} \rightarrow 0$. Thus, applying the estimate in (4.20) from Lemma 4.1, we conclude that

$$
\lim _{\delta \downarrow 0}\left(F_{\delta}^{\tilde{\varepsilon}}, \varphi_{\beta, \delta}\right)=\left(F^{\tilde{\varepsilon}}, \varphi_{\beta}\right) .
$$

Finally, the proof of (iii) is done using the argument from Lemma (4.3).

\section{Renormalization for Higher-Order Moments}

In the previous sections, we focused on the asymptotic behavior of $\left\langle T\left(\frac{x}{\delta}, \frac{y}{\delta}, \frac{t}{\varrho^{2}(\delta)}\right)\right\rangle$ as $\delta \rightarrow 0$ and specifically on the crossovers in the scaling laws and effective equations obtained by varying the spectral parameters $\tilde{\varepsilon}$ and $z$. While the first moment of $T_{\delta}$ is a natural quantity describing macroscopic properties of Eq. (1.1), it is also important to investigate the limits of higher order moments of solutions,

$$
\left\langle\left(T\left(\frac{x}{\delta}, \frac{y}{\delta}, \frac{t}{\varrho^{2}(\delta)}\right)\right)^{n}\right\rangle, \quad n=2,3,4,
$$

in cases of infrared-divergent velocity fields. In this section we compute explicitly the limits (6.1) for model problem (4.1) using function space integrals and scaling arguments involving the exact form of the energy spectrum. Similar formulas are true in the case of time-dependent velocity fields but for simplicity in exposition, we do not describe those results here. We find a sharp contrast at the level of convergence of moments, between the mean field regimes and the anomalous regimes in which long range convective effects are dominant. In fact, in the latter cases we have

$$
\lim _{\delta \downarrow}\left\langle\left[T\left(\frac{x}{\delta}, \frac{y}{\delta}, \frac{t}{\varrho^{2}(\delta)}\right)\right]^{n}\right\rangle \neq(\bar{T}(x, y, t))^{n}, \text { for } n \geqq 2,
$$

while, in the mean field regime the functions $T\left(\frac{x}{\delta}, \frac{y}{\delta}, \frac{t}{\varrho^{2}(\delta)}\right)$ converge uniformly in $(x, y, t)$ to $\bar{T}(x, y, t)$, for almost all realizations (see the appendix) so that there is an identity in (6.2).

For simplicity we restrict the calculations to the time independent case, focusing on the anomalous regimes $0<\tilde{\varepsilon}<2$ and $2 \leqq \tilde{\varepsilon}<4$. Recall that the scaling 
function $\varrho$ satisfies

$$
\varrho(\delta)=\left\{\begin{array}{lll}
\frac{1}{\delta^{1+\varepsilon / 2}}, & \text { for } & 0<\tilde{\varepsilon}<2 \\
\delta^{1 / 2}\left(\log \frac{1}{\delta}\right)^{1 / 4}, & \text { for } \quad & \tilde{\varepsilon}=2 \\
\delta^{1-\tilde{\varepsilon} / 4}, & \text { for } & 2<\tilde{\varepsilon}<4,
\end{array}\right.
$$

and, in particular $\delta / \varrho(\delta) \ll 1$, so that the solution of (4.1) satisfies, as $\delta \downarrow 0$,

$$
T\left(\frac{x}{\delta}, \frac{y}{\delta}, \frac{t}{\varrho^{2}(\delta)}\right)=\frac{1}{2 \pi} \iint \mathscr{E}\left[x, \xi, v_{\delta}, \beta, t\right] e^{i(x \eta+y \xi)} \widehat{T}_{0}(\xi, \eta) d \xi d \eta+o(1),
$$

where

$$
\mathscr{E}\left[x, \xi, v_{\delta}, \beta, t\right]=E\left\{\exp \left[i \xi \delta \int_{0}^{t / e^{2}} v_{\delta}\left(\frac{x}{\delta}+\beta(s)\right) d s\right]\right\} .
$$

Let $n$ be a positive integer, $n \geqq 2$. In order to compute the $n^{\text {th }}$ order moment of $T$, we introduce $n$ independent Brownian motions $\beta_{j}(t), 1 \leqq j \leqq n$. Using (6.4), we can write

$$
\begin{aligned}
{\left[T\left(\frac{x}{\delta}, \frac{y}{\delta}, \frac{t}{\varrho^{2}(\delta)}\right)\right]^{n}=} & \left.\left(\frac{1}{2 \pi}\right)^{n} \int_{\mathbf{R}^{2 n}} \mathscr{E}_{\delta}^{(n)}\left[x, \xi, v_{\delta}, \boldsymbol{\beta}, t\right] e^{i\left(x \sum_{j} n_{j}+y \sum_{j} \xi_{j}\right.}\right) \\
& \times \widehat{\mathbf{T}}_{0}^{(n)}(\xi, \boldsymbol{\eta}) d \xi d \boldsymbol{\eta}+o(1),
\end{aligned}
$$

where $\xi=\left(\xi_{1}, \ldots, \xi_{n}\right), \boldsymbol{\eta}=\left(\eta_{1}, \ldots, \eta_{n}\right), \boldsymbol{\beta}=\left(\beta_{1}, \ldots, \beta_{n}\right)$,

$$
\mathscr{E}_{\delta}^{(n)}\left[x, \xi, v_{\delta}, \boldsymbol{\beta}, t\right]=E\left[e^{\left.i t^{\prime e^{2}} \int_{0}^{\int} j_{j=1}^{n}\left(\xi_{j} v_{\delta}\right) x / \delta+\beta_{j}(s)\right) d s}\right],
$$

and

$$
\hat{\mathbf{T}}_{0}^{(n)}(\xi, \eta)=\prod_{j=1}^{n} \hat{T}_{0}\left(\xi_{i}, \eta_{i}\right)
$$

Taking ensemble average over the Gaussian velocity statistics (2.11) and using (3.10), we obtain an asymptotic formula for the averaged $n^{\text {th }}$ moment as $\delta \rightarrow 0$ :

$$
\begin{aligned}
& \left\langle\left[T\left(\frac{x}{\delta}, \frac{y}{\delta}, \frac{t}{\varrho^{2}}\right)\right]^{n}\right\rangle=\left(\frac{1}{2 \pi}\right)^{n} \int_{\mathbf{R}^{2 n}}\left\langle\mathscr{E}_{\delta}^{(n)}\left[x, \xi, v_{\delta}, \boldsymbol{\beta}, t\right]\right\rangle \\
& \times e^{i\left(\underset{j}{x \sum_{j} \eta_{j}+y \sum_{j} \xi_{j}}\right)} \widetilde{T}_{0}(\xi, \boldsymbol{\eta}) d \xi d \boldsymbol{\eta}+o(1) .
\end{aligned}
$$

It is therefore necessary to evaluate the limit

$$
\begin{aligned}
\lim _{\delta \downarrow 0} & \left\langle\mathscr{E}_{\delta}^{(n)}\left[x, \xi, v_{\delta}, \boldsymbol{\beta}, t\right]\right\rangle \\
& =\lim ^{\delta \downarrow 0}\left\langle E\left[e^{j \sum^{n} i \xi_{j} \delta^{t / \varrho^{2}} v_{\delta}} \frac{x}{\delta}+v^{1 / 2} \beta_{j}(s) d s\right]\right\rangle \\
& =\lim _{\delta \downarrow 0} E\left\{\exp \left[-\sum_{j, k=1}^{n} \xi_{j} \xi_{k} \frac{\delta^{2}}{2} \int_{0}^{t / \varrho^{2}} \int_{0}^{t / \varrho^{2}} R_{\delta}\left(v_{0}^{1 / 2} \beta_{j}(s)-v_{0}^{1 / 2} \beta_{k}\left(s^{\prime}\right)\right) d s d s\right]\right\} \\
& =\lim _{\delta \downarrow 0} E\left\{\exp \left[-\sum_{j, k=1}^{n} \xi_{j} \xi_{k} \delta^{2} \varrho^{-4} \frac{t^{2}}{2} \int_{0}^{1} \int_{0}^{1} R_{\delta}\left[\frac{v_{0}^{1 / 2} t^{1 / 2}}{\varrho}\left(\widetilde{\beta}_{j}(s)-\widetilde{\beta}_{k}\left(s^{\prime}\right)\right)\right] d s d s^{\prime}\right]\right\} .
\end{aligned}
$$


To proceed with this evaluation, we consider separately the regimes $2 \leqq \tilde{\varepsilon}<4$ and $0<\tilde{\varepsilon}<2$.

A) The Hyperscaling Regime $2 \leqq \tilde{\varepsilon}<4$. From the results in Sect. 4B), we chose the scaling function as

$$
\varrho(\delta)=\left\{\begin{array}{lll}
\delta^{1 / 2}\left(\log \frac{1}{\delta}\right)^{1 / 4} & \text { if } & \tilde{\varepsilon}=2 \\
\delta^{1-\tilde{\varepsilon} / 4} & \text { if } & 2<\tilde{\varepsilon}<4 .
\end{array}\right.
$$

With such a choice, it is easily seen that, for all $\boldsymbol{\beta}$,

$$
\lim _{\delta \downarrow 0} \delta^{2} \varrho^{-4} \int_{0}^{1} \int_{0}^{1} R_{\delta}\left[\frac{\left(v_{0} t\right)^{1 / 2}}{\varrho}\left(\widetilde{\beta}_{j}(s)-\widetilde{\beta}_{k}\left(s^{\prime}\right)\right)\right] d s d s^{\prime}=D(\tilde{\varepsilon}), \quad 1 \leqq j, k \leqq n,
$$

where

$$
D(\tilde{\varepsilon})= \begin{cases}\frac{1}{2 \pi}, & \text { if } \quad \tilde{\varepsilon}=2 \\ \frac{1}{2 \pi} \int \psi_{0}(|k|)|k|^{1-\tilde{\varepsilon}} d k, & 2<\tilde{\varepsilon}<4 .\end{cases}
$$

Applying this result to (6.10), we conclude that

$$
\lim _{\delta \downarrow 0}\left\langle\mathscr{E}_{\delta}^{(n)}\left[x, \xi, v_{\delta}, \boldsymbol{\beta}, t\right]\right\rangle=e^{-\frac{t^{2}}{2} D(\xi)\left(\sum_{j=1}^{n} \xi_{j}\right)^{2}},
$$

and hence that

$$
\begin{aligned}
& \lim _{\delta \downarrow 0}\left\langle\left[T\left(\frac{x}{\delta}, \frac{y}{\delta}, \frac{t}{\varrho^{2}}\right)\right]^{n}\right\rangle \\
& \quad=\frac{1}{(2 \pi)^{n} / 2} \int_{\mathbf{R}^{n}} e^{-\frac{t^{2}}{2} D(\xi)\left(\Sigma_{j} \xi_{j}\right)^{2}} \prod_{j=1}^{n}\left[e^{i \xi_{j} y} \widehat{T}_{0}^{(y)}\left(x, \xi_{j}\right)\right] d \xi,
\end{aligned}
$$

where

$$
\hat{T}_{0}^{(y)}(x, \xi)=\frac{1}{\sqrt{2 \pi}} \int_{-\infty}^{+\infty} e^{i y \xi} T_{0}(x, y) d y
$$

is the Fourier transform of the initial data $T_{0}$ in the variable $y$. It is not hard to derive an evolution equation for

$$
\left.\bar{T}_{n}(x, y, t) \equiv \lim _{\delta \downarrow 0}\left\langle T\left(\frac{x}{\delta}, \frac{y}{\delta}, \frac{t}{\varrho^{2}}\right)\right)^{n}\right\rangle
$$

from the integral equation (6.15). In fact, since

$$
\frac{d}{d y} \prod_{j=1}^{n} e^{i \xi_{j} y} \widehat{T}_{0}^{(y)}\left(x, \xi_{j}\right)=i\left(\sum_{j=1}^{n} \xi_{j}\right) \prod_{j=1}^{n} e^{i \xi_{j} y} \widehat{T}_{0}^{(y)}\left(x, \xi_{j}\right)
$$


it follows that for $n=1,2, \ldots, \bar{T}_{n}$ satisfies the same diffusion equation

$$
\frac{\partial \bar{T}_{n}}{\partial t}=t D(\tilde{\varepsilon}) \frac{\partial^{2}}{\partial y^{2}} \bar{T}_{n}, \quad t>0,
$$

with initial conditions $\bar{T}_{n}(t=0)=\left(T_{0}(x, y)\right)^{n}$. We remark that this fact immediately implies a stronger result, namely that, for all $\Phi \in C_{0}(\mathbf{R})$, and all $T_{0}(x, y) \in C_{0}^{\infty}(\mathbf{R})$, setting

$$
\overline{\Phi(\mathrm{T})}(\mathrm{x}, \mathrm{y}, \mathrm{t}) \equiv \lim _{\delta \downarrow 0}\left\langle\Phi\left(\frac{x}{\delta}, \frac{y}{\delta}, \frac{t}{\varrho^{2}(\delta)}\right)\right\rangle
$$

we have

$$
\frac{\overline{\partial \Phi(T)}}{\partial t}=t D(\tilde{\varepsilon}) \frac{\partial^{2}}{\partial y^{2}} \overline{\Phi(T)},\left.\quad \overline{\Phi(T)}\right|_{t=0}=\Phi\left(T_{0}(x, y)\right) .
$$

Remark. This result can be readily reinterpreted in the language of weak convergence involving Young measures $([29,30])$. We develop this remark in detail elsewhere but mention here that the density of the Young measure at value $\lambda$ is given by

$$
\frac{d \tilde{\mu}}{d \lambda}(x, y, t)=\frac{1}{\sqrt{2 \pi t^{2} D(\tilde{\varepsilon})}} \int_{-\infty}^{+\infty} d \bar{y} e^{-\frac{|y-\bar{y}|^{2}}{2 D(\varepsilon) t^{2}}} \delta\left(\lambda-T_{0}(x, \bar{y})\right),
$$

where $\boldsymbol{\delta}(\cdot)$ is the Dirac delta function. In particular, since the Young measure is not a Dirac mass, genuine weak convergence occurs and there is no equality in (6.2) in general. The fact that the limits of all moments $\bar{T}_{n}(x, y, t)$ and the functions $\Phi\left(T\left(\frac{x}{\delta}, \frac{y}{\delta}, \frac{t}{\varrho^{2}}\right)\right)$ satisfy the same effective equation as $\delta \rightarrow 0$ is intuitively obvious if one draws the parallel with inviscid dynamics (cf. Sect. 4) replacing the diffusion equation by

$$
\frac{\partial T}{\partial t}+v_{\delta}(x) \frac{\partial T}{\partial y}=0 .
$$

We obtain, multiplying both sides of the equation by $T^{n-1}$,

$$
\frac{\partial T^{n}}{\partial t}+v_{\delta}(x) \frac{\partial T^{n}}{\partial y}=0
$$

so that the limit functions $\overline{T_{n}}$ should satisfy, heuristically, the same limiting equation. Thus, (6.21) is a manifestation of the analogy of the model problem with the corresponding "inviscid" problem with $v_{0}=0$ in the regime $2 \leqq \varepsilon<4$ as we developed in Sect. 4.

B) The Hyperscaling Regime, $0<\tilde{\varepsilon}<2$. The calculation of $\overline{T_{n}}(x, y, t)$ for $0<\tilde{\varepsilon}<2$ is more subtle due to the nontrivial role played by the Brownian motions $\widetilde{\beta}_{1}, \widetilde{\beta}_{2}, \ldots, \widetilde{\beta}_{n}$ in (6.10). For $n \geqq 2$, it is convenient to define the random measures

$$
\mu_{i j}(d x)=\operatorname{mes}\left\{\left(s, s^{\prime}\right) \in[0,1]^{2}: x \leqq \widetilde{\beta}_{i}(s)-\widetilde{\beta}_{j}\left(s^{\prime}\right) \leqq x+d x\right\}
$$


for $1 \leqq i, j \leqq n$. In particular, the measures $\mu_{i i}(d x) 1 \leqq i \leqq n$ coincide with the measure $\mu_{\beta}(d x)$ defined in Sect. 4 to study the limit as $\delta \rightarrow 0$ of the rescaled first moment of $T$.

Returning to Eq. (6.10), we observe that for each $j, l, 1 \leqq j, l \leqq n$, we have

$$
\int_{0}^{1} \int_{0}^{1} R_{\delta}\left[\frac{v_{0}^{1 / 2} t^{1 / 2}}{\varrho}\left(\widetilde{\beta}_{j}(s)-\widetilde{\beta}_{l}\left(s^{\prime}\right)\right)\right] d s d s^{\prime}=\int_{-\infty}^{+\infty} R_{\delta}\left[\frac{v_{0}^{1 / 2} t^{1 / 2}}{\varrho} x\right] \mu_{j}(d x)
$$

so that the Wiener integral on the right-hand side of (6.10) can be rewritten as

$$
E\left\{\exp \left[\sum_{j, l=1}^{n} \xi_{j} \xi_{l} \delta^{2} \varrho^{-4} \frac{t^{2}}{2} \int_{-\infty}^{+\infty} R_{\delta}\left[\frac{v_{0}^{1 / 2} t^{1 / 2}}{\varrho} x\right] \mu_{j l}(d x)\right]\right\}
$$

with the scaling function $\varrho=\varrho(\delta)$ given by

$$
\varrho(\delta)=\delta^{\frac{1}{1+\tilde{\varepsilon} / 2}}, \quad 0<\tilde{\varepsilon}<2 .
$$

We wish to take the limit as $\delta \rightarrow 0$ of the multiple Wiener integral (6.24). The terms $\int R_{\delta}\left[\frac{v_{0}^{1 / 2} t^{1 / 2}}{\varrho} x\right] \mu_{j l}(d x)$ are functionals of the paths $\widetilde{\beta}_{j}(s), \widetilde{\beta}_{l}(s), 1 \leqq j, l \leqq n$, and the strategy outlined in Sect. 4, Lemmas (4.1) and (4.2) applies provided that the conditions

$$
\frac{d \mu_{j l}}{d x} \in H^{3 / 2-\gamma} \cap F e^{-1}\left(L^{\infty}\right), \quad \gamma>0, \quad 1 \leqq j, l \leqq n,
$$

on the densities of the off-diagonal measures $\mu_{j l}, j \neq l$ hold. We have already proved that (6.26) is valid for $j=l$, for a measure involving a single path. To check condition (6.26) for $j \neq l$ it suffices to take $j=1, l=2$ and to consider the identity:

$$
\hat{\mu}_{12}(k)=(2 \pi)^{-1 / 2}\left(\int_{0}^{1} e^{i k \tilde{\beta}_{1}(s)} d s\right)\left(\int_{0}^{1} e^{i k \tilde{\beta}_{2}(s)} d s\right) .
$$

In particular, $\left\|\hat{\mu}_{12}\right\|_{L^{\infty}(\mathscr{R})} \leqq 1$. Using the inequality $|a b| \leqq \frac{1}{2}\left(|a|^{2}+|b|^{2}\right)$, we have

$$
\begin{aligned}
\left|\hat{\mu}_{12}(k)\right| & \leqq \frac{1}{2}(2 \pi)^{-1 / 2}\left|\int_{0}^{1} e^{i k \tilde{\beta}_{1}(s)} d s\right|^{2}+\frac{1}{2}(2 \pi)^{-1 / 2}\left|\int_{0}^{1} e^{i k \tilde{\beta}_{2}(s)} d s\right|^{2} \\
& =\frac{1}{2} \hat{\mu}_{11}(k)+\frac{1}{2} \hat{\mu}_{22}(k) .
\end{aligned}
$$

We conclude that, for fixed $\widetilde{\beta}_{1}, \widetilde{\beta}_{2}$,

$$
\begin{aligned}
\int\left(1+|k|^{2}\right)^{3 / 2-\gamma}\left|\hat{\mu}_{12}(k)\right|^{2} d k \leqq & \frac{1}{2} \int\left(1+|k|^{2}\right)^{3 / 2-\gamma}\left|\hat{\mu}_{11}(k)\right|^{2} d k \\
& +\frac{1}{2} \int\left(1+|k|^{2}\right)^{3 / 2-\gamma}\left|\hat{\mu}_{22}(k)\right|^{2} d k,
\end{aligned}
$$

so that, by Lemma (4.2),

$$
\varphi_{12}(x) \underset{\text { Def }}{\equiv} \frac{d \mu_{12}(x)}{d x} \in H^{3 / 2-\gamma} \cap F e^{-1}\left(L^{\infty}\right), \quad \boldsymbol{\beta} \text { a.e. },
$$

as desired. 
Using the arguments of Lemma (4.1), we obtain

$$
\begin{aligned}
\lim _{\delta \downarrow 0} t^{2} \delta^{2} \varrho^{-4} \int R_{\delta}\left(\frac{v_{0}^{1 / 2} t^{1 / 2}}{\varrho} x\right) d \mu_{j l}(d x) & =t^{1+\tilde{z} / 2} v_{0}^{\tilde{z} / 2-1}(2 \pi)^{-1 / 2}\left(F^{\tilde{\varepsilon}}, \frac{d \mu_{j l}}{d x}\right) \\
& =t^{1+\xi / 2} v_{0}^{\tilde{z} / 2-1}(2 \pi)^{1 / 2} \sigma_{j, l}^{2}(\boldsymbol{\beta}),
\end{aligned}
$$

where

$$
\sigma_{j, l}^{2}(\beta)=\left\{\begin{array}{cc}
C(\tilde{\varepsilon}) \int \frac{\varphi_{j l}(x)-\varphi_{j l}(0)}{|x|^{2-\tilde{\varepsilon}}} d x, & 0<\tilde{\varepsilon}<1, \\
C(1) \varphi_{j l}(0), & \tilde{\varepsilon}=1, \\
C(\tilde{\varepsilon}) \int \frac{\varphi_{j l}(x) d x}{|x|^{2-\tilde{\varepsilon}}}, 1<\tilde{\varepsilon}<2, &
\end{array}\right.
$$

with

$$
\varphi_{j l}(x)=\frac{d \mu_{j l}(x)}{d x},
$$

and with $C(\tilde{\varepsilon})$ as in (4.17). Thus, Eq. (6.10) becomes,

$$
\lim _{\delta \downarrow 0}\left\langle\mathscr{E}_{\delta}^{(n)}\left[x, \xi, v_{\delta} \xi, t\right]\right\rangle=E\left\{\exp \left[\sum_{j, k=1}^{n} \xi_{j} \xi_{k} \frac{t^{1+\tilde{z} / 2}}{2} v_{0}^{\tilde{z} / 2-1}(2 \pi)^{-1 / 2} \sigma_{j k}^{2}(\boldsymbol{\beta})\right]\right\} .
$$

Substitution of this formula in (6.9) and passage to the limit yields the final expression

$$
\begin{aligned}
\bar{T}_{n}(x, y, t)= & \lim _{\delta \downarrow 0}\left\langle\left[T\left(\frac{x}{\delta}, \frac{y}{\delta}, \frac{t}{\varrho^{2}(\delta)}\right)\right]^{n}\right\rangle \\
= & \int_{\mathbf{R}^{n}} E\left\{\exp \left[-\frac{t^{1+z / 2}}{2} v_{0}^{z / 2-1}(2 \pi)^{-1 / 2} \sum_{j k=1}^{n} \sigma_{j k}^{2}(\boldsymbol{\beta}) \xi_{j} \xi_{k}\right]\right\} \\
& \times e^{i y \Sigma} \xi_{j}^{n} \prod_{j=1}^{n} \hat{T}_{0}^{y}\left(x, \xi_{j}\right) d \xi .
\end{aligned}
$$

As in the case of the first moment, for the regime $0<\tilde{\varepsilon}<2$, the equation satisfied by $\bar{T}_{n}$ is not local, due to the randomness of the "diffusivities" $\sigma_{j k}^{2}(\boldsymbol{\beta}), 1 \leqq j, k \leqq n$. We note finally that the expression (6.35) can be recast as a multiple convolution integral

$$
\bar{T}_{n}(x, y, t)=\int_{\mathbf{R}^{n}}\left[\int \underline{v}(d \underline{\sigma}) K\left(t^{1+\varepsilon / 2}, y-\bar{y}_{1}, \ldots, y-\bar{y}_{n} ; \sigma^{2}\right)\right] \prod_{j=1}^{n} T_{0}\left(x, \bar{y}_{j}\right) d \overline{\mathbf{y}},
$$

where

$$
K\left(t, y_{1} \ldots y_{n}\right)=\frac{\left(\operatorname{Det} \underline{\sigma}^{2}\right)^{-1 / 2}}{(2 \pi)^{n / 2} D(\tilde{\varepsilon})^{n / 2}} \exp \left\{-\frac{D(\tilde{\varepsilon})}{2} \mathbf{y}^{T}\left[\underline{\sigma}^{2}\right]^{-1} \mathbf{y}\right\}
$$

with $\underline{\sigma}^{2}=\left(\sigma_{j, k}^{2}\right)$ and

$$
D(\tilde{\varepsilon})=t v_{0}^{z / 2-1}(2 \pi)^{-1 / 2}
$$


The measure $\underline{y}(d \sigma)$ is the appropriate generalization of the distribution function involving random diffusivities as described in Sect. 4.

\section{Appendix: The Mean-Field Theory}

For velocity statistics of type I, i.e. $\tilde{\varepsilon}<\max \{0,2-z\}$, the behavior is diffusive. The corresponding averaging theorems for the partial differential equations satisfied by the scalar $T^{\delta}\left(\frac{x}{\delta}, \frac{t}{\delta^{2}}\right)$ in the long-time/large-scale limit, can be derived using a method for homogenizing equations with random coefficients developed by Papanicolaou and Varadhan $([14,26,27])$. For completeness' sake we include the proofs of these results for our model problem. To the authors' knowledge, our results on the time-dependent case are new. The above-mentioned references should be consulted for further details on homogenization.

a) Time-independent velocity statistics with $\tilde{\varepsilon}<0$.

From (3.5) the computation of the effective Green's function reduces to finding the value of

$$
\lim _{\delta \downarrow 0} E\left[e^{\left.i \delta Z\left(\frac{t}{\delta^{2}}\right)\right]},\right.
$$

where

$$
Z(t)=\eta v_{0}^{1 / 2} \beta(t)-\xi \int_{0}^{t} v_{\delta}\left(v_{0}^{1 / 2} \beta(s)\right) d s
$$

The strategy for computing this limit is to decompose $\delta Z\left(\frac{t}{\delta^{2}}\right)$ as

$$
\delta Z\left(\frac{t}{\delta^{2}}\right)=\delta I_{\delta}\left(\frac{t}{\delta^{2}}\right)+R_{\delta}(t),
$$

where $I_{\delta}(\cdot)$ is a suitable stochastic integral and $R_{\delta}(t)$ is a remainder that converges to zero in probability as $\delta \rightarrow 0$. The evaluation of these limits in (A.2) will follow from an application of the ergodic theorem.

Step 1 . We construct an auxiliary function, or corrector, $X_{\delta}(x)$ defined by

where

$$
X_{\delta}(x)=2 \int \frac{e^{i k x}-1}{v_{0} k^{2}} d \mu_{\delta}(k),
$$

$$
d \mu_{\delta}(k)=\frac{1}{\sqrt{2 \pi}}|k|^{\frac{1-\tilde{\varepsilon}}{2}}\left(\psi_{0}\left(\frac{k}{\delta}\right)\right)^{1 / 2}\left(\psi_{\infty}(k)\right)^{1 / 2} d W(k) .
$$

This function has the following properties:

(i)

$$
\left\langle\mathrm{X}_{\delta}^{2}(\mathrm{x})\right\rangle=\frac{2}{\pi} \int \frac{\left|\mathrm{e}^{i k x}-1\right|^{2}}{v_{0}^{2} \mathrm{k}^{4}}|\mathrm{k}|^{1-\tilde{\varepsilon}} \psi_{0}\left(\frac{k}{\delta}\right) \psi_{\infty}(k) d k<\infty,
$$


so $X_{\delta}(x)$ is a well-defined Gaussian process, with the estimate

$$
\left\langle\left|X_{\delta}(x)\right|^{2}\right\rangle \leqq \frac{2 x^{2}}{\pi v_{0}^{2}} \int|k|^{-1-\tilde{\varepsilon}^{2}} \psi_{\infty}(k) d k \leqq C x^{2}
$$

(ii) The derivative

$$
X_{\delta}^{\prime}(x)=\frac{2 i}{v_{0}} \int e^{i k x} k^{-1} d \mu_{\delta}(k)
$$

is a stationary Gaussian process with variance

$$
\left\langle\left|X_{\delta}^{\prime}(x)\right|^{2}\right\rangle=\frac{4}{v_{0}^{2}} \int \frac{\left\langle\left|d \mu_{\delta}(k)\right|^{2}\right\rangle}{k^{2}}=\frac{2 x^{2}}{\pi v_{0}^{2}} \int|k|^{-(1+\varepsilon)} \psi_{0}\left(\frac{k}{\delta}\right) \psi_{\infty}(k) d k,
$$

and

(iii)

$$
X_{\delta}^{\prime \prime}(x)=-\frac{2}{v_{0}} v_{\delta}(x)
$$

Applying Itô's formula [19] to $X_{\delta}\left(v_{0}^{1 / 2}(\beta(s))\right)$, we obtain

$$
\left.\int_{0}^{t} v_{\delta}\left(v_{0}^{1 / 2} \beta(s)\right)\right) d s=v_{0}^{1 / 2} \int_{0}^{t} X_{\delta}^{\prime}\left(v_{0}^{1 / 2}(\beta(s))\right) d \beta(s)-\left[X_{\delta}\left(v_{0}^{1 / 2} \beta(t)\right)-X_{\delta}(0)\right],
$$

and hence, from (A.5),

$$
\delta Z\left(\frac{t}{\delta^{2}}\right)=v_{0}^{1 / 2} \delta \int_{0}^{t / \delta^{2}}\left[\eta-\xi X_{\delta}^{\prime}\left(v_{0}^{1 / 2} \beta(s)\right)\right] d \beta(s)+R_{\delta}(t)=\delta I\left(\frac{t}{\delta^{2}}\right)+R_{\delta}(t),
$$

where

$$
I(t)=v_{0}^{1 / 2} \int_{0}^{t}\left[\eta-\xi X_{\delta}^{\prime}\left(v_{0}^{1 / 2} \beta(s)\right)\right] d \beta(s)
$$

and

$$
R_{\delta}(t) \equiv \delta X_{\delta}\left[v_{0}^{1 / 2} \beta\left(\frac{t}{\delta^{2}}\right)\right]-\delta X_{\delta}(0)
$$
Equation (A.6) is the desired decomposition of the exponent $\delta Z\left(\frac{t}{\delta^{2}}\right)$. We now
estimate the remainder $R_{\delta}(t)$.

Step 2. We claim that

$$
\lim _{\delta \downarrow 0} R_{\delta}(t)=0
$$

in probability. To see this, note that

$$
\left\langle\delta^{2} X_{\delta}^{2}\left(\frac{x}{\delta}\right)\right\rangle=\frac{4 \delta^{2}}{v_{0}^{2}} \int \frac{\left|e^{i k x / \delta}-1\right|^{2}}{k^{2}} \frac{\left\langle\left|d \mu_{\delta}(k)\right|^{2}\right\rangle}{k^{2}}=\frac{4 \delta^{2}}{v_{0}^{2}} \int \frac{\sin ^{2}\left(\frac{k x}{2 \delta}\right)}{k^{2}} d m_{\delta}(k),
$$


where

$$
d m_{\delta}(k)=\frac{1}{2 \pi}|k|^{-1-\tilde{\varepsilon}} \psi_{0}\left(\frac{k}{\delta}\right) \psi_{\infty}(k) d k
$$

Hence,

$$
\left\langle\left|\delta^{2} X_{\delta}^{2}\left(\frac{x}{\delta}\right)\right|\right\rangle \leqq \frac{2 \delta^{2}}{\pi v_{0}^{2}} \int \frac{\sin ^{2}\left(\frac{k x}{2 \delta}\right)}{k^{2}}|k|^{-1-\tilde{\varepsilon}} \psi_{\infty}(k) d k
$$

and, since $|k|^{-1-\tilde{\varepsilon}} \psi_{\infty}(k) \in L^{1}(\mathbf{R})$,

$$
\lim _{\delta \downarrow 0|x| \leqq M} \sup _{\delta \downarrow}\left\langle\delta^{2} X_{\delta}^{2}\left(\frac{x}{\delta}\right) \bigvee \leqq \frac{2 M^{2}}{\pi v_{0}^{2}} \lim _{\delta \rightarrow 0} \int \frac{\sin ^{2}(k / \delta)}{(k / \delta)^{2}}|k|^{-(1-\tilde{\varepsilon})} \psi_{\infty}(k) d k=0 .\right.
$$

Next we show that the decay of $\delta^{2} X_{\delta}^{2}\left(\beta\left(\frac{t}{\delta^{2}}\right)\right)$ can be established, using the estimate in (A.8) and the fact that Brownian motion has exponentially small tails. In fact,

$$
\begin{aligned}
E^{\beta}\left(\left\langle\delta^{2} X_{\delta}^{2}\left(\beta\left(\frac{t}{\delta^{2}}\right)\right)\right\rangle\right) & =E^{\tilde{\beta}}\left(\left\langle\delta^{2} X_{\delta}^{2}\left(\frac{\widetilde{\beta}(t)}{\delta}\right)\right\rangle\right) \\
& =\frac{1}{\sqrt{2 \pi t}} \int_{-\infty}^{+\infty}\left\langle\delta^{2} X_{\delta}^{2}\left(\frac{x}{\delta}\right)\right\rangle e^{-\frac{x^{2}}{2 t}} d x \\
& \leqq \sup _{|k| \leqq M}\left\langle\delta^{2} X_{\delta}^{2}\left(\frac{x}{\delta}\right)\right\rangle+\frac{C}{\sqrt{2 \pi t}} \int_{|x| \geqq M} x^{2} e^{-\frac{x^{2}}{2 t}} d x,
\end{aligned}
$$

where (A.3) was used. Hence, for all $M$

$$
\varlimsup_{\delta \downarrow 0} E^{\beta}\left(\left\langle\delta^{2} X_{\delta}^{2}\left(\beta\left(\frac{t}{\delta^{2}}\right)\right)\right\rangle\right) \leqq \frac{C}{\sqrt{2 \pi t}} \int_{|x| \geqq M} x^{2} e^{-\frac{x^{2}}{2 t}} d x .
$$

We conclude from this that for $M$ sufficiently large,

$$
\varlimsup_{\delta \downarrow 0} E\left\{\left\langle\delta^{2} X_{\delta}^{2}\left(\beta\left(\frac{t}{\delta^{2}}\right)\right)\right\rangle\right\} \leqq C_{1} e^{-\frac{M}{4 t}},
$$

where $C_{1}$ is a numerical constant. Letting $M$ tend to zero,

$$
\lim _{\delta \downarrow 0} \delta X_{\delta}\left(\beta\left(\frac{t}{\delta^{2}}\right)\right)=0 \text { in probability } .
$$

Step 3. We have shown that $\delta Z\left(t / \delta^{2}\right)$ and $\delta I_{\delta}\left(t / \delta^{2}\right)$ have the same limiting distributions. This common limiting distribution can be evaluated using the fact that $\left\{I_{\delta}\left(\cdot / \delta^{2}\right)\right\}$ for $\delta \leqq 1$ is a family of continuous-time martingales with quadratic variations:

$$
Q_{\delta}(s)=\frac{1}{\delta^{2}} \int_{0}^{s / \delta^{2}} v_{0}\left[\eta-\xi^{2} X_{\delta}^{\prime}\left(v_{0}^{1 / 2} \beta(\tau)\right)\right]^{2} d \tau, \quad 0 \leqq \mathrm{~s} \leqq \mathrm{t}
$$


which satisfy

$$
\begin{aligned}
\left\langle Q_{\delta}(t)\right\rangle^{2} & =\frac{1}{\delta^{2}} \int_{0}^{t / \delta^{2}} v_{0}\left\langle\left[\eta-\xi X_{\delta}^{\prime}(0)\right]^{2}\right\rangle d s \\
& =t v_{0}\left[\eta^{2}+\xi^{2}\left\langle\left|X_{\delta}^{\prime}(0)\right|^{2}\right\rangle\right] \\
& \leqq t v_{0}\left[\eta^{2}+\frac{2 \xi^{2}}{\pi v_{0}^{2}} \int|k|^{-1-\varepsilon^{2}} \psi_{\infty}(k) d k\right]
\end{aligned}
$$
By a standard martingale theory argument $[11] \delta I_{\delta}\left(\frac{t}{\delta^{2}}\right)$ converges weakly to a
martingale with quadratic variation

$$
\bar{Q}(s)=\lim _{\delta \rightarrow 0} Q_{\delta}(s), \quad 0 \leqq s \leqq t .
$$

To compute this limit, we first remove the infrared cutoff $\psi_{0}\left(\frac{k}{\delta}\right)$ by defining

$$
X_{0}(x)=\frac{1}{\sqrt{2 \pi}} \int \frac{e^{i k x}-1}{k^{2}}|k|^{(1-\tilde{\varepsilon}) / 2} \psi_{\infty}^{1 / 2}(k) d W(k)
$$

and

$$
\widetilde{Q}_{\delta}(s)=\frac{1}{2} \int_{0}^{s / \delta^{2}} v_{0}\left[\eta-\xi X_{0}^{\prime}\left(v_{0}^{1 / 2} \beta(\tau)\right)\right]^{2} d \tau
$$

It can easily be checked that

$$
\lim _{\delta \downarrow 0} E\left\langle\left|Q_{\delta}(t)-\widetilde{Q}_{\delta}(t)\right|\right\rangle=0,
$$

so the infrared cutoff $\psi_{0}\left(\frac{k}{\delta}\right)$ is negligible in this regime. To compute $\lim _{\delta \downarrow 0} \widetilde{Q}_{\delta}(t)$, we note that the real-valued process $X(t)=X_{0}^{\prime}\left(v_{0}^{1 / 2} \beta(t)\right)$ has an invariant probability measure $\varrho(d x) \equiv \operatorname{Pr}\left\{X_{0}^{\prime} \in d x\right\}$ and is ergodic relative to time translations. This last fact follows from the fact that $\beta(t)$ visits all points and that $X_{0}^{\prime}(x)$ is ergodic under spatial translations. From the ergodic theorem, we conclude that

$$
\begin{aligned}
\bar{Q}(s) & =\lim _{\delta \downarrow 0} \tilde{Q}_{\delta}(s)=s v_{0}^{2}\left\langle\left|\eta-\xi X_{0}^{\prime}\right|^{2}\right\rangle \\
& =v_{0} s\left[\eta^{2}+\frac{2 \xi^{2}}{\pi v_{0}^{2}} \int|k|^{-1-\tilde{\varepsilon}} \psi_{\infty}(k) d k\right] .
\end{aligned}
$$

Thus, $\delta I_{\delta}\left(\cdot / \delta^{2}\right)$ converges weakly to a continuous-time martingale with quadratic variation $\bar{Q}(s)$, i.e. a Wiener process. In particular, $\delta I_{\delta}\left(t / \delta^{2}\right)$ converges in distribution to a normal random variable with variance

$$
\bar{Q}(t)=v_{0} t\left[\eta^{2}+\frac{2 \xi^{2}}{\pi v_{0}^{2}} \int|k|^{-1-\varepsilon_{\infty}} \psi_{\infty}(k) d k\right] .
$$

Equivalently, the enhanced effective diffusivity in the $y$ direction is given by

$$
v^{*}=v_{0}+\frac{2}{\pi v_{0}} \int|k|^{-1-\tilde{\varepsilon}} \psi_{0}(k) d k
$$


b) Time-dependent statistics with $0<z \leqq 2$ and $\tilde{\varepsilon}<\max (0,2-z)$.

In the time-dependent case, diffusive behavior can be shown using a similar technique. We outline the main parts of the corresponding three-step argument, focussing on the treatment of the singularity of the spectrum in the infrared $(|k| \ll 1)$ region. The basic result is that the solution of the initial-value problem

$$
\begin{gathered}
\frac{\partial T^{\delta}}{\partial t}+\frac{1}{\delta} v_{\delta}\left(\frac{x}{\delta}, \frac{t}{\delta^{2}}\right) \frac{\partial T^{\delta}}{\partial y}=\frac{1}{2} v_{0} \Delta T^{\delta}, \quad t>0, \\
\left.T^{\delta}\right|_{t}=0=T_{0}(x, y)
\end{gathered}
$$

converges in probability as $\delta \rightarrow 0$ to the solution of the (deterministic) equation

$$
\frac{\partial \bar{T}}{\partial t}=\frac{1}{2} v_{0} \frac{\partial^{2} \bar{T}}{\partial x^{2}}+\frac{1}{2} v^{*} \frac{\partial^{2} \bar{T}}{\partial y^{2}},\left.\quad \bar{T}\right|_{t=0}=T_{0}(x)
$$

with

$$
v^{*}=v_{0}+\frac{2}{\pi} \int \frac{|k|^{1-\varepsilon} \psi_{\infty}(k) d k}{v_{0} k^{2}+2 a|k|^{2}} .
$$

From the arguments of Sect. 3, involving the Feynman-Kac formula, this result reduces to the calculation of

$$
\lim _{\delta \downarrow 0} E\left[e^{\delta Z_{\delta}\left(\frac{t}{\delta^{2}}\right)}\right],
$$

where

$$
Z_{\delta}(t)=v_{0}^{1 / 2} \eta \beta(t)-\xi \int_{0}^{t} v_{\delta}\left(v_{0}^{1 / 2} \beta(s), t-s\right) d s .
$$

Following the procedure of the previous section, we set

$$
d \mu_{\delta}(k, \omega)=\frac{1}{\sqrt{2 \pi}}|k|^{\frac{1-\tilde{\varepsilon}}{2}} \psi_{0}^{1 / 2}\left(\frac{k}{\delta}\right) \psi_{\infty}^{1 / 2}(k)\left(a|k|^{2}\right)^{-1 / 2}\left(\Phi\left(\frac{\omega}{a|k|^{2}}\right)\right)^{1 / 2} d W(k, \omega),
$$

and

$$
d m_{\delta}(k, \omega)=\frac{1}{2 \pi}|k|^{1-\tilde{\varepsilon}} \psi_{0}\left(\frac{k}{\delta}\right) \psi_{\infty}(k)\left(a|k|^{2}\right)^{-1} \Phi\left(\frac{\omega}{a|k|^{2}}\right) d k d \omega,
$$

with $\Phi(s)=\pi^{-1}\left(1+s^{2}\right)^{-1}$; we define the corrector $X_{\delta}$ by

$$
X_{\delta}(x, t)=2 \iint \frac{\left(e^{i k x+i \omega t}-1\right)}{2 i \omega+v_{0} k^{2}} d \mu_{\delta}(k, \omega) .
$$

It can be readily checked that $X_{\delta}(x, t)$ is well-defined. Moreover,

$$
\frac{\partial X_{\delta}(x, t)}{\partial x}=2 i \int \frac{e^{i k x-i \omega t} k}{2 i \omega+v_{0} k^{2}} d \mu(k, \omega)
$$


is a stationary process, with variance

$$
\begin{aligned}
& \left\langle\left|\frac{\partial X_{\delta}}{\partial x}(0,0)\right|^{2}\right\rangle=\frac{2}{\pi} \int \frac{k^{2} d m(k, \omega)}{4 \omega^{2}+v_{0}^{2} k^{4}} \\
& =\frac{2}{\pi^{2}} \int|k|^{1-\tilde{\varepsilon}} \psi_{0}\left(\frac{k}{\delta}\right) \psi_{\infty}(k) d k \int \frac{k^{2}}{4 \omega^{2}+v_{0}^{2} k^{4}} \cdot \frac{\left(a|k|^{z}\right)^{-1}}{1+\left(\frac{\omega}{a|k|^{z}}\right)^{2}} d \omega \\
& =\frac{1}{v_{0}} \int|k|^{1-\tilde{\varepsilon}} \psi_{0}\left(\frac{k}{\delta}\right) \psi_{\infty}(k) d k \int e^{-|t|\left(\frac{v_{0} k^{2}}{2}+a|k|^{2}\right)} d t \\
& =\frac{2}{\pi v_{0}} \int \frac{|k|^{1-\tilde{\varepsilon}} \psi_{0}\left(\frac{k}{\delta}\right) \psi_{\infty}(k) d k}{v_{0}|k|^{2}+2 a|k|^{z}} .
\end{aligned}
$$

Note that this integral converges independently of whether the infrared cutoff $\psi_{0}\left(\frac{k}{\delta}\right)$ is present or not since the strength of the singularity is $|k|^{1-\tilde{\varepsilon}-\min (2, z)}$ and we are in the regime $\tilde{\varepsilon}<\max (2,2-z)$. This corrector satisfies the equation

$$
\left(\frac{\partial}{\partial t}-\frac{v_{0}}{2} \frac{\partial^{2}}{\partial x^{2}}\right) X_{\delta}(x, t)=v_{\delta}(x, t)
$$

Applying Itô's formula to $X_{\delta}\left(v_{0}^{1 / 2} \beta(t), t-s\right)$, we obtain

$$
\begin{aligned}
\int_{0}^{t} v_{\delta}\left(v_{0}^{1 / 2} \beta(s), t-s\right) d s= & v_{0}^{1 / 2} \int_{0}^{t} \frac{\partial X_{\delta}}{\partial x}\left(v_{0}^{1 / 2} \beta(s), t-s\right) d s \\
& -X_{\delta}\left(v_{0}^{1 / 2} \beta(t), 0\right)+X_{\delta}(0, t),
\end{aligned}
$$

and thus, from the definition of $Z_{\delta}(t)$, in (A.9),

$$
\delta Z\left(\frac{t}{\delta^{2}}\right)=v_{0}^{1 / 2} \delta \int_{0}^{t / \delta^{2}}\left[\eta-\xi \frac{\partial X}{\partial x}\left(v_{0}^{1 / 2} \beta(s), \frac{t}{\delta^{2}}-s\right)\right] d \beta(s)+R_{\delta}(t)
$$

where

$$
R_{\delta}(t)=\delta X_{\delta}\left[v_{0}^{1 / 2} \beta\left(\frac{t}{\delta^{2}}\right), 0\right]-\delta X_{\delta}\left(0, t / \delta^{2}\right) .
$$

The second step of the proof consists in showing that $R_{\delta}(t)$ converges to zero in probability. To determine the behavior of $\delta X_{\delta}\left(v_{0}^{1 / 2} \beta\left(\frac{t}{\delta^{2}}\right), 0\right)$, we make the
preliminary estimate $\left\langle\delta^{2} X^{2}\left(\frac{x}{\delta}, 0\right)\right\rangle \leqq \frac{|x|^{2}}{v_{0}} \iint \frac{\sin ^{2}\left(\frac{k x}{2 \delta}\right)}{\left(\frac{k x}{2 \delta}\right)^{2}} \cdot \frac{k^{2} d \mu(k, \omega)}{4 \omega^{2}+v_{0} k^{4}} \leqq \frac{|x|^{2}}{v_{0}} \int \frac{\sin ^{2}\left(\frac{k x}{2 \delta}\right)}{\left(\frac{k x}{2 \delta}\right)^{2}} \cdot \frac{|k|^{1-\varepsilon} \psi_{\infty}(k) d k}{v_{0} k^{2}+2 a|k|^{2}}$. 
Since $|k|^{1-\tilde{\varepsilon}} \psi_{\infty}(k)\left[v_{0}|k|^{2}+2 a|k|^{2}\right]^{-1}$ is integrable, by the dominated convergence theorem,

$$
\lim _{\delta \rightarrow 0} \sup _{|x| \leqq M}\left\langle\delta^{2} X_{\delta}^{2}\left(\frac{x}{\delta}, 0\right)\right\rangle \leqq \frac{M^{2}}{\pi v_{0}} \lim \delta \frac{\sin ^{2}(k / \delta)|k|^{1-\tilde{\varepsilon}} \psi_{\infty}(k) d k}{(k / \delta)^{2} v_{0}|k|^{2}+2 a|k|^{2}}=0 .
$$

This fact implies that

$$
\lim _{\delta \downarrow 0} \delta X_{\delta}\left[v_{0}^{1 / 2} \beta\left(\frac{t}{\delta^{2}}\right), 0\right]=0
$$

in probability, by an argument similar to the one used in the time-independent case, see (A.6). The second summand in (A.14) satisfies

$$
\begin{aligned}
\left\langle\delta^{2} X_{\delta}^{2}\left(0, \frac{t}{\delta^{2}}\right)\right\rangle & =4 \delta^{2} \iint \frac{\left|e^{i \omega t / \delta^{2}}-1\right|^{2}}{4 \omega^{2}+v_{0} k^{4}} d m_{\delta}(k, \omega) \\
& \leqq 8 \delta^{2} \iint \frac{\left|e^{i \omega t / \delta^{2}}-1\right|}{4 \omega^{2}+v_{0}^{2} k^{4}} d m_{\delta}(k, \omega) \\
& \leqq \frac{C_{1} t}{v_{0}} \iint\left|\frac{\sin \left(\omega t / 2 \delta^{2}\right)}{\omega t / 2 \delta^{2}}\right| \frac{|\omega|}{|\omega|^{2}+k^{4}} d m_{\delta}(k, \omega)
\end{aligned}
$$

where $C_{1}$ is a suitable numerical constant. We now check that the density of the measure

$$
\frac{|\omega|}{\omega^{2}+k^{4}} d m_{\delta}(k, \omega)
$$

is dominated by an integrable function, thus guaranteeing the convergence of

$$
\left\langle\delta^{2} X_{\delta}\left(0, \frac{t}{\delta^{2}}\right)\right\rangle
$$

to zero. In fact, the above-mentioned density is dominated by

$$
\frac{1}{\pi} \frac{|\omega|}{\omega^{2}+k^{4}} \cdot \frac{\left(a|k|^{z}\right)^{-1}}{1+\left(\frac{\omega}{a|k|^{z}}\right)^{2}}|k|^{1-z^{2}} \psi_{\infty}(k)
$$

and we have

$$
\begin{aligned}
& \frac{1}{\pi} \iint \frac{|\omega|}{\omega^{2}+k^{4}} \frac{\left(a|k|^{z}\right)^{-1}}{1+\left(\frac{\omega}{a|k|^{2}}\right)^{2}}|k|^{1-\tilde{\varepsilon}} \psi_{\infty}(k) d k d \omega \\
& \quad=\frac{1}{\pi} \int|k|^{1-\tilde{\varepsilon}} \psi_{\infty}(k) d k \int \frac{|\omega| k^{2}}{1+\left(\frac{\omega}{k^{2}}\right)^{2}} \frac{1}{k^{2}} \cdot \frac{\left(a|k|^{z}\right)^{-1}}{\left(1+\frac{\omega}{a|k|^{z}}\right)^{2}} d \omega .
\end{aligned}
$$




\section{Setting}

$$
f(t) \equiv \int_{-\infty}^{+\infty} \frac{|s|}{1+s^{2}} e^{i s t} d s
$$

we note that $f(t) \in L^{2}(\mathbf{R})$. By Plancherel's theorem the integral in (A.16) is estimated as follows:

$$
\begin{aligned}
& \int|k|^{1-\tilde{\varepsilon}} \psi_{\infty}(k) d k \int \frac{|\omega| / k^{4}}{1+\frac{\omega^{2}}{k^{4}}} \cdot \frac{\left(a|k|^{z}\right)^{-1}}{1+\left(\frac{\omega}{a k^{2}}\right)^{2}} d \omega \\
& \quad \leqq \frac{1}{\pi} \int|k|^{1-\tilde{\varepsilon}} \psi_{\infty}(k) d k\left(\int f\left(k^{2} t\right) e^{-a|k|^{z} t} d t\right) \\
& \quad \leqq \frac{1}{\pi} \int|k|^{1-\tilde{\varepsilon}} \psi_{\infty}(k) d k\left(\int f^{2}\left(k^{2} t\right) d t\right)^{1 / 2}\left(\int e^{-2 a|k| z t} d t\right)^{1 / 2} \\
& \quad \leqq C \int|k|^{1-\tilde{\varepsilon}} \psi_{\infty}(k)|k|^{-1}|k|^{-z / 2} d k<\infty,
\end{aligned}
$$

since the singularity strength is $|k|^{-\tilde{\varepsilon}-z / 2}$ and $\tilde{\varepsilon}<\max \{0,2-z\}$. Having established that $\delta Z_{\delta}\left(t / \delta^{2}\right)$ and the stochastic integral

$$
\delta \int_{0}^{t / \delta^{2}} v_{0}^{1 / 2}\left[\eta-\xi \frac{\partial X}{\partial x}\left(v_{0}^{1 / 2} \beta(s), \frac{t}{\delta^{2}}-s\right)\right] d \beta(s)
$$

have the same limiting distribution as $\delta \rightarrow 0$, the final step consists in computing this distribution. As in the time-independent case, this can be done using a martingale-theory argument and the ergodic theorem. Note that (A.17) is the value at $s=t$ of the martingale

$$
\delta \int_{0}^{s / \delta^{2}} v_{0}^{1 / 2}\left[\eta-\xi \frac{\partial X}{\partial x}\left(v_{0}^{1 / 2} \beta(\tau), \frac{t}{\delta^{2}} \tau\right)\right] d \beta(\tau)
$$

which has quadratic variation

$$
Q_{\delta}(s)=\delta^{2} \int_{0}^{s / s^{2}} v_{0}\left[\eta-\xi \frac{\partial X_{\delta}}{\partial x}\left(v_{0}^{1 / 2} \beta(\tau), \frac{t}{\delta^{2}}-\tau\right)\right]^{2} d \tau
$$

As in the previous section, by the ergodic theorem

$$
\lim _{\delta \downarrow 0} Q_{\delta}(s)=v_{0} S\left[\eta^{2}+\frac{2 / \pi \xi^{2}}{v_{0}} \int \frac{|k|^{1-\tilde{\varepsilon}} \psi_{\infty}(k) d k}{v_{0}|k|^{2}+2 a|k|^{z}}\right],
$$

in probability. Thus the stochastic integral (A.18) converges in distribution to a normal random variable with variance

$$
v_{0} t\left[\eta^{2}+\frac{2 / \pi \xi^{2}}{v_{0}} \int \frac{|k|^{1-\tilde{\varepsilon}} \psi_{\infty}(k) d k}{v_{0}|k|^{2}+2 a|k|^{2}}\right] .
$$

This proves our claim; the enhanced diffusivity in the $y$-direction being

$$
v^{*}=v_{0}+2 / \pi \int \frac{|k|^{1-\tilde{\varepsilon}} \psi_{\infty}(k) d k}{v_{0}|k|^{2}+2 a|k|^{2}} .
$$




\section{References}

1. Adams, R.: Sobolev spaces. New York: Academic Press 1975

2. Avellaneda, M., Majda, A.: Approximate and exact renormalization theories for a model for turbulent transport (in preparation)

3. Avellaneda, M., Majda, A.: On the long-time, large scale renormalized diffusivity for turbulent transport (submitted to Physica D)

4. Batchelor, G.K.: The theory of homogeneous turbulence. Cambridge Science Classics, Cambridge: Cambridge University Press 1982

5. Bensoussan, A., Lions, J.L., Papanicolaou, G.: Asymptotics analysis for periodic structures. Amsterdam: North Holland 1978

6. Chorin, A.J.: Math. Comp. 27, 1-15 (1973)

7. Csanady, G.T.: Turbulent diffusion in the environment. Geophysics and Astrophysics Monographs. Dordrecht, Holland: D. Reidel 1973

8. Forster, D., Nelson, D., Stephen, M.: Phys. Rev. A 16, 732-749 (1977)

9. Gelfand, I.M., Vilenkin, N.Ya.: Generalized functions IV; applications of harmonic analysis. New York, London: Academic Press 1964

10. Gelfand, I.M., Silov, G.: Generalized functions, Vol. 1. New York: Academic Press 1964

11. Helland, I.S.: Scand. J. Stat. g, 79-94 (1982)

12. Hitzl, D., Maltz, F.: J. Comp. Phys. 37, 218-241 (1980)

13. Koch, D., Brady, J.: Phys. Fluids A 1, 47-51 (1989)

14. Koch, D., Brady, J.: J. Fluid Mech. 180, 387-403 (1987)

15. Kraichnan, R.: Phys. Fluids 6, 575-598 (1965)

16. Kraichnan, R.: Complex Systems 1, 805-820 (1987)

17. Martin, P.C., De Dominicis, C.: Suppl. Prog. Theor. Phys. 64, 108-123 (1978)

18. Matheron, G., De Marsily, G.: Water Resources Res. 16, 901-917 (1980)

19. McKean, H.P.: Stochastic integrals. New York, London: Academic Press 1969

20. McLaughlin, D., Papanicolaou, G., Pironneau, O.: SIAM J. Appl. Math. 45, 780-807 (1985)

21. Oehlschläger, K.: Ann. Prob. 16, 1084-1126 (1988)

22. Osada, H.: Proceedings 4th Jap.-USSR Symp. Prob. Theory. Lecture Notes in Mathematics, vol. 1021, pp. 507-517. Berlin, Heidelberg, New York: Springer 1983

23. Papanicolaou, G., Varadhan, S.R.S.: Boundary Value Problems with Rapidly Oscillating Coefficients. In: Random Fields, Colloq. Math. Soc. Janos Bolyai, 27. Fritz, J., Kebowitz, J.L., Szasz, D. (eds.) pp. 835-873. Amsterdam: North-Holland (1982)

24. Roberts, P.H.: J. Fluid Mech. 11, 257-273 (1961)

25. Rose, H.A.: J. Fluid Mech. 81, 719-734 (1977)

26. Taylor, G.I.: Proc. Lond. Math. Soc. Ser. 2 20, 196-203 (1921)

27. Yakhot, V., Orszag, S.: Analysis of the $\varepsilon$-Expansion in Turbulence Theory: Approximate Renormalization Group for Diffusion of a Passive Scalar in a Random Velocity Field (preprint: October, 1988)

28. Zeldovich, Ya.B.: Sov. Phys. Dokl. 27, 797-799 (1982)

29. Tartar, K.: Compensated compactness and applications to partial differential equations. In: Nonlinear analysis and mechanics. Heriot-Watt Symposium, IV, pp. 136-192, Research Notes in Math. London: Pitman

30. Diperna, R.J., Majda, A.J.: Oscillations and Concentrations in Weak Solutions of the Incompressible Fluid Equations. Commun. Math. Phys. 108, 667-689 (1987)

Communicated by A. Jaffe

Received December 6, 1989 
